\title{
LIMIT THEOREMS FOR NUMBERS OF MULTIPLE RETURNS IN NONCONVENTIONAL ARRAYS
}

\author{
YURI KIFER \\ INSTITUTE OF MATHEMATICS \\ HEBREW UNIVERSITY \\ JERUSALEM, ISRAEL
}

\begin{abstract}
For a $\psi$-mixing process $\xi_{0}, \xi_{1}, \xi_{2}, \ldots$ we consider the number $\mathcal{N}_{N}$ of multiple returns $\left\{\xi_{q_{i, N}(n)} \in \Gamma_{N}, i=1, \ldots, \ell\right\}$ to a set $\Gamma_{N}$ for $n$ until either a fixed number $N$ or until the moment $\tau_{N}$ when another multiple return $\left\{\xi_{q_{i, N}(n)} \in \Delta_{N}, i=1, \ldots, \ell\right\}$ takes place for the first time where $\Gamma_{N} \cap \Delta_{N}=\emptyset$ and $q_{i, N}, i=1, \ldots, \ell$ are certain functions of $n$ taking on nonnegative integer values when $n$ runs from 0 to $N$. The dependence of $q_{i, N}(n)$ 's on both $n$ and $N$ is the main novelty of the paper. Under some restrictions on the functions $q_{i, N}$ we obtain Poisson distributions limits of $\mathcal{N}_{N}$ when counting is until $N$ as $N \rightarrow \infty$ and geometric distributions limits when counting is until $\tau_{N}$ as $N \rightarrow \infty$. We obtain also similar results in the dynamical systems setup considering a $\psi$-mixing shift $T$ on a sequence space $\Omega$ and studying the number of multiple returns $\left\{T^{q_{i, N}(n)} \omega \in A_{n}^{a}, i=1, \ldots, \ell\right\}$ until the first occurrence of another multiple return $\left\{T^{q_{i, N}(n)} \omega \in A_{m}^{b}, i=1, \ldots, \ell\right\}$ where $A_{n}^{a}, A_{m}^{b}$ are cylinder sets of length $n$ and $m$ constructed by sequences $a, b \in \Omega$, respectively, and chosen so that their probabilities have the same order.
\end{abstract}

\section{INTRODUCTION}

In [13] we considered nonconventional arrays of the form

$$
S_{N}=\sum_{n=1}^{N} \prod_{j=1}^{\ell} T^{q_{j, N}(n)} f_{j}
$$

where $T$ is a measure preserving transformation on a probability space $(\Omega, \mathcal{F}, P)$, $f_{j}$ 's are bounded measurable functions and $q_{j, N}(n)=p_{j} n+q_{j} N, j=1, \ldots, \ell$ are nonnegative functions with integer $p_{j}$ and $q_{j}$ 's. It was shown there that when $p_{j}$ 's are distinct and $T$ is weakly mixing then $\frac{1}{N} S_{N} \rightarrow \prod_{1 \leq j \leq \ell} \int f_{j} d P$ as $N \rightarrow \infty$ in $L^{2}$. Without the weak mixing assumption this convergence fails, in general, even along sufficiently dense subsequences as the following example due to Frantzikinakis shows. Namely, take $\ell=2, q_{1, N}(n)=n+N, q_{2, N}(n)=2 n, f_{1}(x)=e^{4 \pi i x}, f_{2}(x)=$ $e^{-2 \pi i x}$ and $T: \mathbb{S}^{1} \rightarrow \mathbb{S}^{1}$ having the form $T e^{2 \pi i y}=e^{2 \pi i(y+\alpha)}$ for an irrational $\alpha$. Then $\frac{1}{N} S_{N}=e^{2 \pi i(x+2 N \alpha)}$ and since $e^{4 \pi i N \alpha}$ visits every arc on $\mathbb{S}^{1}$ with the frequency

Date: October 4, 2019.

2000 Mathematics Subject Classification. Primary: 60F05 Secondary: 37D35, 60J05.

Key words and phrases. Geometric distribution, Poisson distribution, multiple returns, nonconventional sums, $\psi$-mixing, stationary process, shifts. 
proportional to its length there is no convergence of $\frac{1}{N} S_{N}$ as $N \rightarrow \infty$ even along sequences having positive upper density.

The above convergence under weak mixing was a part of the proof in [13] of an extension of the Szemerédi theorem which says that for any subset $\Lambda$ of nonnegative integers with a positive upper density there exists $\varepsilon>0$ and an infinite set $\mathcal{N}_{\Lambda}$ of positive integers with uniformly bounded gaps such that for any $N \in \mathcal{N}_{\Lambda}$ the interval $[0, N]$ contains not less than $\varepsilon N$ integers $n$ with the property that $a_{n}+$ $p_{j} n+q_{j} N \in \Lambda$ for some $a_{n}$ and all $j=0,1, \ldots, \ell$.

An example in [13] showed that when $q_{j, N}(n)$ 's depend polynomially on $n$ and $N$ then weak mixing of $T$ does not suffice for convergence of $\frac{1}{N} S_{N}$ as $N \rightarrow \infty$ even when $\ell=1$ and $q_{1, N}(n)=n N$. On the other hand, it was shown there that if we assume strong $2 \ell$-mixing of $T$ then $\frac{1}{N} S_{N}$ does converge in $L^{2}$ to $\prod_{j=1}^{\ell} f_{j}$ provided the polynomials $q_{1, N}, \ldots, q_{\ell, N}$ are essentially distinct (as polynomials in $n$ and $N$ ) and depend nontrivially on $n$.

These results motivated the study of limit theorems for such nonconventional arrays, among them the strong law of large numbers (convergence almost everywhere and not just in $L^{2}$ ), the central limit theorem and the Poisson limit theorem type results taking also into account that limit theorems for (triangular) arrays is a well studied topic in probability (though it seems to appear rarely in dynamical systems). In Ch. 3 of [11] we derived these types of limit theorems for sums of the form

$$
S_{N}=\sum_{n=0}^{N} F\left(\xi_{q_{1, N}(n)}, \ldots, \xi_{q_{\ell, N}(n)}\right)
$$

where $\xi_{m}, m=0,1, \ldots$ is a sequence of random variables with sufficiently weak dependence, $F$ is a sufficiently regular function and $q_{i, N}(n)=p_{i} n+q_{i} N$ are linear. Under certain mixing conditions we derived almost sure convergence of $\frac{1}{N} S_{N}$ as $N \rightarrow \infty$ assuming only that the integers $p_{i}, i=1, \ldots, \ell$ are nonzero and distinct. On the other hand, the convergence in distribution of $N^{-1 / 2}\left(S_{N}-E S_{N}\right)$ to a normal random variable required that each difference $q_{i}-q_{j}$ must be divisible by the greatest common divisor of $p_{i}$ and $p_{j}$ while without this condition the variance of $N^{-1 / 2}\left(S_{N}-E S_{N}\right)$ may not converge as $N \rightarrow \infty$. For instance, it was shown in [11] that if $\xi_{m}, m=0,1, \ldots$ is an i.i.d. sequence of random variables, $S_{N}=$ $\sum_{n=1}^{N} F\left(\xi_{2 n+N}, \xi_{2 N-2 n}\right)$ with a symmetric function $F$ such that $E F\left(\xi_{0}, \xi_{1}\right)=0$ and $E F^{2}\left(\xi_{0}, \xi_{1}\right)>0$, then $\lim _{N \rightarrow \infty, N \text { odd }} \frac{1}{N} S_{N}^{2} \neq \lim _{N \rightarrow \infty, N \text { even }} \frac{1}{N} S_{N}^{2}$, and so there is no limiting variance which means that the central limit theorem (in the standard form) fails.

Concerning Poisson limit theorems we considered in [11] arrays of the form

$$
S_{N}=\sum_{n=1}^{N} \prod_{j=1}^{\ell} \mathbb{I}_{\Gamma_{N}}\left(\xi_{q_{j, N}(n)}\right)
$$

where $\xi_{m}, m=0,1, \ldots$ is a stationary $\psi$-mixing sequence of random variables, $q_{j, N}(n)=p_{j} n+q_{j} N$ and $\lim _{N \rightarrow \infty} N P\left\{\xi_{0} \in \Gamma_{N}\right\}=\lambda$. Assuming that for any nontrivial permutation $\zeta$ of $(1,2, \ldots, \ell)$ the matrix $\left(\begin{array}{cccc}p_{1} & p_{2} & \ldots & p_{\ell} \\ p_{\zeta(1)} & p_{\zeta(2)} & \ldots & p_{\zeta(\ell)}\end{array}\right)$ has rank 2 we showed that $S_{N}$ converges in distribution to a Poisson random variable while without the above condition this may not hold true, in general. In the dynamical systems setup we obtained under the above condition convergence in distribution 
as $m \rightarrow \infty$ to Poisson random variables of expressions having the form

$$
S_{N_{m}}=\sum_{n=1}^{N_{m}} \prod_{j=1}^{\ell} \mathbb{I}_{A_{m}^{a}} \circ T^{q_{j, N}(n)}
$$

provided that $\lim _{m \rightarrow \infty} N_{m}\left(P\left(A_{m}^{a}\right)\right)^{\ell}=\lambda$, where $q_{j, N}(n)=p_{j} n+q_{j} N, T$ is the left shift on a sequence space $\Omega, A_{m}^{a}$ is a cylinder of length $m$ built by a nonperiodic sequence $a \in \Omega$ and $P$ is a $\psi$-mixing $T$-invariant probability measure on $\Omega$.

An extension of the strong law of large numbers to nonconventional arrays with higher degree essentially distinct polynomials $q_{j, N}(n), j=1, \ldots, \ell$ can be obtained in the same way as in Ch. 3 of 11 just relying on the fact that for any $k$ and $N$ the number of $n$ 's satisfying each equation $q_{i, N}(n)-q_{j, N}(n)=k$ does not exceed the maximum of the degrees of $q_{i, N}$ and $q_{j, N}$. Another proof of this result appears in 9 . The central limit theorem is more complicated and [9] requires that in addition to the above conditions on linear $q_{i}$ 's each pair of nonlinear polynomials $q_{i}, q_{j}$ in $n$ and $N$ with $i \neq j$ satisfies at least one of the two following conditions. The first condition says that for any $\delta>0$ there exist constants $C_{\delta}, N_{\delta}>0$ and sets $\Gamma_{N, \delta} \subset[1, N]$ with cardinality not exceeding $\delta N$ so that nonlinear bivariate polynomials $q_{i}, q_{j}$ with $i \neq j$ satisfy inf $f_{N>N_{\delta}} N^{-1} \min _{m \in[\delta N, N], n \in[\delta N, N] \backslash \Gamma_{N, \delta}}\left|q_{i, N}(m)-q_{j, N}(n)\right|>0$. This condition is clearly satisfied if $q_{i}$ and $q_{j}$ have different degrees as bivariate polynomials. The second condition concerns polynomials with the same degree, say, $\operatorname{deg} q_{i}=\operatorname{deg} q_{j}=k, i \neq j$. Consider the representation $q_{\alpha, N}(n)=\sum_{l=0}^{k} N^{l} Q_{\alpha, l}(y)$, $\alpha=i, j$ where $y=n / N$ and $Q_{\alpha, l}$ 's are nonconstant polynomials with nonnegative integer coefficients whose degree do not exceed $l$. Then the condition requires that $Q_{i, k}(y)=Q_{j, k}\left(c_{i j} y\right)$ and $Q_{i, k-1}(y)-Q_{j, k-1}\left(c_{i j} y\right)=r_{i j} Q_{j, k}^{\prime}\left(c_{i j} y\right)$ for some reals $c_{i j}>0$ and $r_{i j}$. It is clear that this condition is satisfied if $q_{i, N}(n)=q_{i}(n)$ and $q_{j, N}(n)=q_{j}(n)$ are univariate polynomials depending only on $n$.

The present paper is devoted to two related types of limit theorems for nonconventional arrays. The first one is the Poisson type limit theorems as described above but with with a more general class of functions $q_{j, N}(n), j=1, \ldots, \ell$ which include higher degree polynomials in $n$. The second type concerns limit theorems for arrays of the form

$$
S_{N}=\sum_{n=1}^{\tau_{N}} \prod_{j=1}^{\ell} \mathbb{I}_{\Gamma_{N}}\left(\xi_{q_{j, N}(n)}\right),
$$

where $\tau_{N}=\min \left\{k \geq 1: \prod_{j=1}^{\ell} \mathbb{I}_{\Delta_{N}}\left(\xi_{q_{j, N}(n)}\right)\right\}$ with $\Delta_{N}, N \geq 1$ being another sequence of Borel sets. In the dynamical systems setup we have here the sums

$$
S_{N}=\sum_{k=1}^{\tau_{N}} \prod_{j=1}^{\ell} \mathbb{I}_{A_{n_{N}}^{a}} \circ T^{q_{j, N}(k)},
$$

where $\tau_{N}=\min \left\{k \geq 1: \prod_{j=1}^{\ell} \mathbb{I}_{A_{m_{N}}^{b}} \circ T^{q_{j, N}(k)}=1\right\}$ with $b$ being another nonperiodic sequence and $n_{N}, m_{N} \rightarrow \infty$ as $N \rightarrow \infty$. It turns out that if $P\left\{\xi_{0} \in \Delta_{N}\right\} / P\left\{\xi_{0} \in \Gamma_{N}\right\}$ and $P\left(A_{n_{N}}^{a}\right) / P\left(A_{m_{N}}^{b}\right)$ are bounded away from zero and infinity then under certain conditions $S_{N}$ converges in distribution to a random variable having the geometric distribution. Observe that even for $q_{j, N}(n)$ depending just on $n$ our results generalize and specify somewhat both [14] (which improved the results from [12]) and [15] while the additional dependence on $N$ brings here additional peculiarities. 
Our results remain valid for dynamical systems possessing appropriate symbolic representations such as Axiom A diffeomorphisms (see [5]), expanding transformations and some maps having symbolic representations with an infinite alphabet and $\psi$-mixing invariant measure such as the Gauss map with its Gauss invariant measure and more general $f$-expansions (see [10]). A direct application of the above results in the symbolic setup yields the corresponding results for arrivals to elements of Markov partitions but employing additional technique (see, for instance, [16) it is not difficult to extend these results for arrivals to shrinking geometric balls.

\section{Preliminaries and Main Results}

2.1. $\psi$-mixing processes. Let $(\Omega, \mathcal{F}, P)$ be a probability space and $\mathcal{F}_{m n}, 0 \leq m \leq$ $n \leq \infty$ be a two parameter family of $\sigma$-algebras $\mathcal{F}_{m n}, 0 \leq m \leq n \leq \infty$ such that $\mathcal{F}_{m n} \subset \mathcal{F}_{m^{\prime} n^{\prime}} \subset \mathcal{F}$ provided that $m^{\prime} \leq m \leq n \leq n^{\prime}$. Recall, that the $\psi$-dependence coefficient between two $\sigma$-algebras $\mathcal{G}$ and $\mathcal{H}$ can be written in the form (see []),

$$
\begin{gathered}
\psi(\mathcal{G}, \mathcal{H})=\sup _{\Gamma \in \mathcal{G}, \Delta \in \mathcal{H}}\left\{\left|\frac{P(\Gamma \cap \Delta)}{P(\Gamma) P(\Delta)}-1\right|, P(\Gamma) P(\Delta) \neq 0\right\} \\
=\sup \left\{\|E(g \mid \mathcal{G})-E(g)\|_{L^{\infty}}: g \text { is } \mathcal{H} \text { - measurable and } E|g| \leq 1\right\}
\end{gathered}
$$

and the $\psi$-dependence (mixing) in the family $\mathcal{F}_{m n}$ is measured by the coefficient

$$
\psi(n)=\sup _{m \geq 0} \psi\left(\mathcal{F}_{0, m}, \mathcal{F}_{m+n, \infty}\right) .
$$

Our first setup includes a $\psi$-mixing identically distributed (not necessarily stationary) sequence of random variables $\xi_{0}, \xi_{1}, \ldots$ defined on $(\Omega, \mathcal{F}, P)$ which means that $\psi(1)<\infty$ and $\psi(n) \rightarrow 0$ as $n \rightarrow \infty$ where $\psi(n)$ is defined by (2.1) with $\mathcal{F}_{m n}=\sigma\left\{\xi_{m}, \ldots, \xi_{n}\right\}$ being the minimal $\sigma$-algebra for which $\xi_{m}, \xi_{m+1}, \ldots, \xi_{n}$ are measurable. We will be counting multiple returns by the sequence $\xi_{0}, \xi_{1}, \ldots$ to measurable sets $\Gamma_{N}$ considering the sum

$$
S_{N}=\sum_{n=1}^{N} \prod_{j=1}^{\ell} \mathbb{I}_{\Gamma_{N}}\left(\xi_{q_{j, N}(n)}\right)
$$

where $q_{1, N}, \ldots, q_{\ell, N}$ are functions in $n$ taking on nonnegative integer values when $0 \leq n \leq N$ and satisfying the conditions below. We will be interested to show, in particular, that $S_{N}$ converges in distribution as $N \rightarrow \infty$ to a Poisson random variable provided that $\lim _{N \rightarrow \infty} N P\left\{\xi_{0} \in \Gamma_{N}\right\}$ exists. A simple example from [11,

$$
S_{2 N}=\sum_{n=0}^{2 N} \mathbb{I}_{\Gamma_{2 N}}\left(\xi_{n}\right) \mathbb{I}_{\Gamma_{2 N}}\left(\xi_{2 N-n}\right)=2 \sum_{n=1}^{N} \mathbb{I}_{\Gamma_{2 N}}\left(\xi_{n}\right) \mathbb{I}_{\Gamma_{2 N}}\left(\xi_{2 N-n}\right)-\mathbb{I}_{\Gamma_{2 N}}\left(\xi_{N}\right)
$$

shows that $S_{2 N}$ can have only even limits when $\lim _{N \rightarrow \infty} N P\left\{\xi_{0} \in \Gamma_{N}\right\}$ exists, and so it cannot converge in distribution as $N \rightarrow \infty$ to a Poisson random variable. Thus, certain restrictions on the polynomials $q_{1, N}, \ldots, q_{\ell, N}$ are needed.

2.1. Assumption. $q_{1, N}(n), \ldots, q_{\ell, N}(n)$ are functions taking on nonnegative integer values on integers $n, N \geq 0$, defined arbitrarily when $n>N$ and such that for some constant $K>0$ and all $N \geq 1$ the following properties hold true:

(i) For any $i \neq j, 1 \leq i, j \leq \ell$ and all integers $k, l$ the numbers of integers $n, 0 \leq n \leq N$ satisfying at least one of the equations

$$
q_{i, N}(n)-q_{j, N}(n)=k \quad \text { and } \quad q_{i, N}(n)=l
$$

do not exceed $K$; 
(ii) For any permutation $\zeta$ of $(1,2, \ldots, \ell)$ the number of pairs $m \neq n$ satisfying $0 \leq m, n \leq N$ and solving the system of equations

$$
q_{i, N}(n)-q_{\zeta(i), N}(m)=0, \quad i=1, \ldots, \ell
$$

does not exceed $K$;

(iii)(stronger than (ii)) The cardinality of the set $D_{N}$ of pairs $m \neq n$ with $0 \leq m, n \leq N$ satisfying

$$
\max _{1 \leq i \leq \ell} q_{i, N}(m) \geq \max _{1 \leq i \leq \ell} q_{i, N}(n) \geq \min _{1 \leq i \leq \ell} q_{i, N}(n) \geq \min _{1 \leq i \leq \ell} q_{i, N}(m)
$$

does not exceed $K$.

Assumption (iii) is clearly stronger than Assumption (ii) and we will need the former in the shifts setup while the latter will be sufficient in the $\psi$-mixing processes setup. Observe, next, that $\ell=2, q_{1, N}(n)=n$ and $q_{2, N}(n)=N-n$ in the example above do not satisfy Assumption 2.1)(ii) since taking the permutation $\zeta(1)=2, \zeta(2)=1$ we see that the system $n-(N-m)=0, N-n-m=0$ has $N+1$ solution pairs $n, N-n$ for $n=0,1, \ldots, N$. Note also that if $\ell=1$ then Assumption 2.1(ii) requires only that for any $N \geq 1$ there exist at most $K$ pairs $n, m, n \neq m$ such that $q_{1, N}(n)=q_{1, N}(m)$. This will hold true if, for instance, there exists $n_{0} \geq 1$ such that $q_{1, N}(n)$ is strictly increasing in $n$ on $\left[n_{0}, \infty\right)$. Furthermore, if $q_{i, N}(n)=r_{i}(n)+g_{i}(N), i=1, \ldots, \ell$ where $r_{i}$ 's are nonconstant, essentially distinct polynomials in $n$ and $g_{i}$ 's are functions of $N$, both nonnegative for $n, N \geq 0$ and taking on integer values on integers, then $q_{i, N}$ 's satisfy Assumption 2.1. Indeed, the number of solutions in Assumption 2.1(i) is bounded by the maximal degree of $r_{i}$ 's. Next, there exists an integer $n_{0} \geq 1$ such that all polynomials $r_{i}, i=1, \ldots, \ell$ are strictly increasing on $\left[n_{0}, \infty\right)$, and so if $m>n \geq n_{0}$ then both $\max _{1 \leq i \leq \ell} q_{i, N}(m)>\max _{1 \leq i \leq \ell} q_{i, N}(n)$ and $\min _{1 \leq i \leq \ell} q_{i, N}(m)>\min _{1 \leq i \leq \ell} q_{i, N}(n)$ which implies Assumption 2.1(iii) (and so Assumption 2.1(ii)).

If $q_{1, N}(n), \ldots, q_{\ell, N}(n)$ are polynomials in $n$ then Assumption 2.1(i) will be satisfied provided $\sup _{N \geq 1} \max _{1 \leq i \leq \ell} \operatorname{deg}_{n} q_{i, N}<\infty$, where $\operatorname{deg}_{n}$ denotes the degree of a polynomial in $n$, since the number of solutions in (2.2) is bounded in this case by $\max \left(\operatorname{deg}_{n} q_{i, N}, \operatorname{deg}_{n} q_{j, N}\right)$. A sufficient condition for Assumption 2.1(ii) to hold true can be obtained with the help of the Bézout theorem (see [18], §2 in Ch. III or [7, Section 11.5) which says that if $f$ and $g$ are two nonconstant bivariate coprime polynomials then the system $f(x, y)=0, g(x, y)=0$ has no more than $\operatorname{deg}(f) \operatorname{deg}(g)$ solution pairs $x, y$. If $f$ and $g$ are not coprime, i.e. there exists a nonconstant polynomial $h=h(x, y)$ such that $f=h \tilde{f}$ and $g=h \tilde{g}$ for some polynomials $\tilde{f}$ and $\tilde{g}$, then each solution of $h=0$ solves also the system $f=0, g=0$, and so the latter system may have infinitely many solutions then. Thus, if for some $N_{0} \geq 1$, each $N \geq N_{0}$ and any nontrivial permutation $\zeta$ of $(1,2, \ldots, \ell)$ there exist $i \neq j$ such that the polynomials $\tilde{q}_{i, N}(n, m)=q_{i, N}(n)-q_{\zeta(i), N}(m)$ and $\tilde{q}_{j, N}(n, m)=q_{j, N}(n)-q_{\zeta(j), N}(m)$ are coprime and nonconstant then Assumption 2.1(ii) holds true provided we can bound uniformly in $N \geq 1$ the number of pairs $m \neq n$ which solve the system $q_{i, N}(n)=q_{i, N}(m)$. For this it suffices to assume that there exists $n_{0} \geq 1$ such that for all $N \geq 1$ each polynomial $q_{i, N}, i=1, \ldots, \ell$ is strictly increasing on $[0, \infty)$.

For any two random variables or random vectors $Y$ and $Z$ of the same dimension denote by $\mathcal{L}(Y)$ and $\mathcal{L}(Z)$ their distribution and by

$$
d_{T V}(\mathcal{L}(Y), \mathcal{L}(Z))=\sup _{G}|\mathcal{L}(Y)(G)-\mathcal{L}(Z)(G)|
$$


the total variation distance between $\mathcal{L}(Y)$ and $\mathcal{L}(Z)$ where the supremum is taken over all Borel sets. Our first result is the following.

2.2. Theorem. Let $\xi_{0}, \xi_{1}, \xi_{2}, \ldots$ be a $\psi$-mixing sequence of identically distributed random variables and assume that Assumptions 2.1(i)-(ii) hold true. Let $\Gamma$ be a Borel set, $X_{n}=X_{n, N}=\prod_{i=1}^{\ell} \mathbb{I}_{\Gamma}\left(\xi_{q_{i, N}(n)}\right)$ and $S_{N}=S_{N}^{\Gamma}=\sum_{n=1}^{N} X_{n}$. Then there exists a constant $C>0$ which does not depend on $\Gamma$ and $N \geq 1$ and such that for any positive integers $M, N, R$,

$$
\begin{gathered}
d_{T V}\left(\mathcal{L}\left(S_{N}^{\Gamma}\right), \operatorname{Pois}\left(\lambda_{N}\right)\right) \leq C\left(N R(\Phi(\Gamma))^{2 \ell}+N M(\Phi(\Gamma))^{\ell+1}\right. \\
\left.+M R(\Phi(\Gamma))^{\ell}+\left(2-(1+\psi(R))^{\ell+1}\right)^{-2} \psi(R)\left(M^{2} \Phi(\Gamma)+N(\Phi(\Gamma))^{\ell}\right)\right),
\end{gathered}
$$

provided $\psi(R)<2^{\frac{1}{\ell+1}}-1$, where $\Phi(\Gamma)=P\left\{\xi_{0} \in \Gamma\right\}, \lambda_{N}=N(\Phi(\Gamma))^{\ell}$ and Pois $(\lambda)$ denotes the Poisson distribution with the parameter $\lambda$.

2.3. Corollary. Under the conditions of Theorem 2.2 suppose that in place of one set $\Gamma$ we have a sequence of Borel sets $\Gamma_{N}$ such that

$$
0<C^{-1} \leq N \Phi\left(\Gamma_{N}\right)^{\ell} \leq C<\infty
$$

for some constant $C$. Then

$$
d_{T V}\left(\mathcal{L}\left(S_{N}^{\Gamma_{N}}\right), \operatorname{Pois}\left(\lambda_{N}\right)\right) \rightarrow 0 \text { as } N \rightarrow \infty
$$

where $\lambda_{N}=N\left(\Phi\left(\Gamma_{N}\right)\right)^{\ell}$. In particular, if

$$
\lim _{N \rightarrow \infty} N\left(\Phi\left(\Gamma_{N}\right)\right)^{\ell}=\lambda
$$

then the distribution of $S_{N}$ converges in total variation as $N \rightarrow \infty$ to the Poisson distribution with the parameter $\lambda$.

Now, let $\Gamma$ and $\Delta$ be disjoint Borel sets and set

$$
\Sigma_{N}^{\Gamma, \Delta}=\sum_{k=1}^{\tau} \prod_{i=1}^{\ell} \mathbb{I}_{\Gamma}\left(\xi_{q_{i, N}(k)}\right)
$$

where $\tau=\tau_{\Delta}=\min \left\{n \geq 1: \xi_{q_{i, N}(k)} \in \Delta\right.$ for $\left.i=1, \ldots, \ell\right\}$ writing $\tau=\infty$ if the set in braces above is empty. Denote also by $\operatorname{Geo}(\rho), \rho \in(0,1)$ the geometric distribution with the parameter $\rho$, i.e.

$$
\operatorname{Geo}(\rho)\{k\}=\rho(1-\rho)^{k} \text { for each } k \in \mathbb{N}=\{0,1, \ldots\} .
$$

Now we can state

2.4. Theorem. Let $\xi_{0}, \xi_{1}, \xi_{2}, \ldots$ be a $\psi$-mixing sequence of identically distributed random variables with a marginal distribution $\Phi$ and assume that Assumptions 2.1(i)-(ii) hold true. Then for any disjoint Borel sets $\Gamma, \Delta$ with $\Phi(\Gamma), \Phi(\Delta)>0$ 
and all positive integers $M, N, R$ with $\psi(R)<2^{\frac{1}{\ell+1}}-1$ we have

$$
\begin{gathered}
d_{T V}\left(\mathcal{L}\left(\Sigma_{N}^{\Gamma, \Delta}\right), G e o(\rho)\right) \leq C\left(\left(1-(\Phi(\Delta))^{\ell}\right)^{N}\right. \\
+N(\Phi(\Gamma)+\Phi(\Delta))^{\ell}\left((1+\psi(M))^{\ell}-1\right)+N R(\Phi(\Gamma)+\Phi(\Delta))^{2 \ell} \\
+N M(\Phi(\Gamma)+\Phi(\Delta))^{\ell+1}+M R(\Phi(\Gamma)+\Phi(\Delta))^{\ell}+M^{2}(\Phi(\Gamma)+\Phi(\Delta)) \\
\left.+\left(2-(1+\psi(R))^{\ell+1}\right)^{-2} \psi(R)\left(N(\Phi(\Gamma)+\Phi(\Delta))^{\ell}+M(\Phi(\Gamma)+\Phi(\Delta))\right)\right)
\end{gathered}
$$

where $\rho=\frac{(\Phi(\Delta))^{\ell}}{(\Phi(\Gamma))^{\ell}+(\Phi(\Delta))^{\ell}}$ and the constant $C>0$ does not depend on $\Phi(\Gamma), \Phi(\Delta)$, $M, N$ and $R$.

Next, let $\Gamma_{N}, \Delta_{N}, N=1,2, \ldots$ be a sequence of pairs of disjoint Borel sets such that

$$
\Phi\left(\Gamma_{N}\right), \Phi\left(\Delta_{N}\right) \rightarrow 0 \text { as } N \rightarrow \infty \text { and } 0<C^{-1} \leq \frac{\Phi\left(\Gamma_{N}\right)}{\Phi\left(\Delta_{N}\right)} \leq C<\infty
$$

for some constant $C$.

2.5. Corollary. Suppose that the conditions of Theorem 2.4 concerning the process $\xi_{0}, \xi_{1}, \xi_{2}, \ldots$ and the polynomials $q_{i, N}(n), i=1, \ldots, \ell$ are satisfied. Let $\Gamma_{N}, \Delta_{N}, N=$ $1,2, \ldots$ be Borel sets satisfying (2.9). Then

$$
d_{T V}\left(\mathcal{L}\left(\Sigma_{N}^{\Gamma_{N}, \Delta_{N}}\right), \operatorname{Geo}\left(\rho_{N}\right)\right) \rightarrow 0 \text { as } N \rightarrow \infty
$$

where $\rho_{N}=\left(\Phi\left(\Gamma_{N}\right)\right)^{\ell}\left(\left(\Phi\left(\Delta_{N}\right)\right)^{\ell}+\left(\Phi\left(\Gamma_{N}\right)\right)^{\ell}\right)^{-1}$. In particular, if

$$
\lim _{N \rightarrow \infty} \frac{\Phi\left(\Delta_{N}\right)}{\Phi\left(\Gamma_{N}\right)}=\lambda
$$

then the distribution of $\Sigma_{N}^{\Gamma_{N}, \Delta_{N}}$ converges in total variation as $N \rightarrow \infty$ to the geometric distribution with the parameter $\left(1+\lambda^{\ell}\right)^{-1}$.

2.2. Shifts. Our second setup consists of a finite or countable set $\mathcal{A}$, the sequence space $\Omega=\mathcal{A}^{\mathbb{N}}$, the $\sigma$-algebra $\mathcal{F}$ on $\Omega$ generated by cylinder sets, the left shift $T: \Omega \rightarrow \Omega$, and a $T$-invariant probability measure $P$ on $(\Omega, \mathcal{F})$. We assume that $P$ is $\psi$-mixing with the $\psi$-dependence coefficient given by (2.1) and (2.2) considered with respect to the $\sigma$-algebras $\mathcal{F}_{m n}, n \geq m$ generated by the cylinder sets $\left\{\omega=\left(\omega_{0}, \omega_{1}, \ldots\right) \in \Omega: \omega_{i}=a_{i}\right.$ for $\left.m \leq i \leq n\right\}$ for some $a_{m}, a_{m+1}, \ldots, a_{n} \in \mathcal{A}$. Clearly, $\mathcal{F}_{m n}=T^{-m} \mathcal{F}_{0, n-m}$ for $n \geq m$. For each word $a=\left(a_{0}, a_{1}, \ldots, a_{n-1}\right) \in \mathcal{A}^{n}$ we will use the notation $[a]=\left\{\omega=\left(\omega_{0}, \omega_{1}, \ldots\right): \omega_{i}=a_{i}, i=0,1, \ldots, n-1\right\}$ for the corresponding cylinder set. Write $\Omega_{P}$ for the support of $P$, i.e.

$$
\Omega_{P}=\left\{\omega \in \Omega: P\left[\omega_{0}, \ldots, \omega_{n}\right]>0 \text { for all } n \geq 0\right\} .
$$

For $n \geq 1$ set $\mathcal{C}_{n}=\left\{[w]: w \in \mathcal{A}^{n}\right\}$. Since $P$ is $\psi$-mixing it follows (see [14, Lemma 3.1) that there exists $v>0$ such that

$$
P(A) \leq e^{-v n} \text { for all } n \geq 1 \text { and } A \in \mathcal{C}_{n} .
$$

For any $n \geq 1$ and $V \in \mathcal{F}_{0, n-1}$ set

$$
\pi(V)=\min \left\{k \geq 1: V \cap T^{-k} V \neq \emptyset\right\}
$$

and $S_{N}^{V}=\sum_{k=1}^{N} \prod_{i=1}^{\ell} \mathbb{I}_{V} \circ T^{q_{i, N}(k)}$. Observe that always $\pi(V) \leq n$ if $V \in \mathcal{F}_{0, n-1}$. 
2.6. Theorem. Suppose that Assumptions 2.1(i) and 2.1(iii) are satisfied. Then there exists a constant $C \geq 1$ such that for any $n, V \in \mathcal{F}_{0, n-1}, N$ and $R$ satisfying $\psi(R-n)<2^{\frac{1}{\ell+1}}-1$ we have,

$$
\begin{gathered}
d_{T V}\left(\mathcal{L}\left(S_{N}^{V}\right), \operatorname{Pois}\left(\lambda_{N}\right)\right) \leq C((R+n) P(V) \\
+\left(n P(V)+N(P(V))^{\ell}\right)\left(R P(V)+\sum_{r=\pi(V)}^{n-1} P\left(T^{n-r} V\right)\right) \\
\left.+N(P(V))^{\ell} \psi(R-n)\left(2-(1+\psi(R-n))^{\ell+1}\right)^{-2}\right)
\end{gathered}
$$

where $\lambda_{N}=N(P(V))^{\ell}$.

2.7. Corollary. Suppose that Assumptions 2.1(i) and 2.1(iii) are satisfied. Let $V_{L} \in \mathcal{F}_{0, n_{L}-1}, L=1,2, \ldots$ be a sequence of sets such that $n_{L} P\left(V_{L}\right) \rightarrow 0$ and $\sum_{r=\pi\left(V_{L}\right)}^{n_{L}-1} P\left(T^{n_{L}-r} V_{L}\right) \rightarrow 0$ as $L \rightarrow \infty$. Let $N_{L} \rightarrow \infty$ as $L \rightarrow \infty$ be a sequence of integers such that $0<C^{-1} \leq \lambda_{L}=N_{L}\left(P\left(V_{L}\right)\right)^{\ell} \leq C<\infty$ for some constant $C$ and all $L \geq 1$. Then

$$
d_{T V}\left(\mathcal{L}\left(S_{N_{L}}^{V_{L}}\right), \operatorname{Pois}\left(\lambda_{L}\right)\right) \rightarrow 0 \text { as } L \rightarrow \infty
$$

and if $\lim _{L \rightarrow \infty} \lambda_{L}=\lambda$ then the distribution of $S_{N_{L}}^{V_{N_{L}}}$ converges in total variation as $L \rightarrow \infty$ to the Poisson distribution with the parameter $\lambda$. In particular, if $V_{L}=A_{n_{L}}^{\eta}=\left[\eta_{0}, \ldots, \eta_{n_{L}-1}\right]=\left\{\omega \in \Omega: \omega_{0}=\eta_{0}, \ldots, \omega_{n_{L}-1}=\eta_{n_{L}-1}\right\}$ with $n_{L} \rightarrow \infty$ as $L \rightarrow \infty$ and $\eta \in \Omega_{P}$ is nonperiodic then $\pi\left(A_{n_{L}}^{\eta}\right) \rightarrow \infty$ as $L \rightarrow \infty$ and the above statements hold true for such $V_{L}$ 's provided the above conditions on $\lambda_{L}$ are satisfied.

Next, for any $V \in \mathcal{F}_{0, n-1}, V \neq \emptyset$ and $W \in \mathcal{F}_{0, m-1}, W \neq \emptyset$ define

$$
\pi(V, W)=\min \left\{k \geq 1: V \cap T^{-k} W \neq \emptyset \text { or } W \cap T^{-k} V \neq \emptyset\right\} .
$$

It is clear that $\pi(V, W) \leq m \wedge n$, and so

$$
\kappa_{V, W}=\min \{\pi(V, W), \pi(V), \pi(W)\} \leq m \wedge n
$$

where, as usual, for $n, m \geq 1$ we denote $m \wedge n=\max \{m, n\}$ and $m \vee n=\min \{m, n\}$. Set

$$
\tau_{W}(\omega)=\min \left\{k \geq 1: T^{q_{i, N}(k)} \omega \in W \text { for } i=1, \ldots, \ell\right\}
$$

with $\tau_{W}(\omega)=\infty$ if the event in braces does not occur and define

$$
\Sigma_{N}^{V, W}=\sum_{k=1}^{\tau_{W}} \prod_{i=1}^{\ell} \mathbb{I}_{V} \circ T^{q_{i, N}(k)} .
$$

2.8. Theorem. Assume that Assumptions 2.1](i) and 2.1(iii) are satisfied. Then there exists a constant $C>0$ such that for any disjoint sets $V \in \mathcal{F}_{0, n-1}$ and $W \in \mathcal{F}_{0, m-1}$ with $P(V), P(W)>0$ and all integers $n, m, N, R \geq 1$ satisfying $\psi(R-n \vee m)<2^{\frac{1}{\ell+1}}-1$ we have

$$
\begin{aligned}
& d_{T V}\left(\mathcal{L}\left(\Sigma_{N}^{V, W}\right), G e o(\rho)\right) \leq C\left(\left(1-(P(W))^{\ell}\right)^{N}+(n \vee m)(P(V)+P(W))\right. \\
& +N(P(V)+P(W))^{\ell}\left((1+\psi(n \vee m))^{\ell}-1+\psi(R-n \vee m)\right. \\
& +R(P(V)+P(W))+\sum_{r=\kappa_{V, W}}^{n \vee m-1}\left(P\left(T^{n \vee m-1} V\right)+\left(P\left(T^{n \vee m-1} W\right)\right)\right)
\end{aligned}
$$

where $\rho=\frac{(P(W))^{\ell}}{(P(V))^{\ell}+(P(W))^{\ell}}$. 
2.9. Corollary. Suppose that Assumptions 2.1(i) and 2.1(iii) hold true. Let $V_{L} \in$ $\mathcal{F}_{0, n_{L}-1}$ and $W_{L} \in \mathcal{F}_{0, m_{L}-1}, L=1,2, \ldots$ be two sequences of sets such that

$$
\begin{gathered}
\left(n_{L} \vee m_{L}\right)\left(P\left(V_{L}\right)+P\left(W_{L}\right)\right) \rightarrow 0 \quad \text { as } L \rightarrow \infty, \\
\alpha_{L}=\sum_{r=\kappa_{V_{L}, W_{L}}}^{n_{L} \vee m_{L}-1}\left(P\left(T^{n_{L} \vee m_{L}-r} V_{L}\right)+P\left(T^{n_{L} \vee m_{L}-r} W_{L}\right)\right) \rightarrow 0 \text { as } L \rightarrow \infty
\end{gathered}
$$

and for some constant $C$ and all $L \geq 1$,

$$
0<C^{-1} \leq \frac{P\left(V_{L}\right)}{P\left(W_{L}\right)} \leq C<\infty .
$$

Let $N_{L}, L=1,2, \ldots$ be a sequence satisfying

$$
N_{L}\left(P\left(W_{L}\right)\right)^{\ell} \rightarrow \infty \text { and }
$$

Then

$$
d_{T V}\left(\mathcal{L}\left(\Sigma_{N_{L}}^{V_{L}, W_{L}}\right), \operatorname{Geo}\left(\rho_{L}\right)\right) \rightarrow 0 \text { as } L \rightarrow \infty
$$

where $\rho_{L}=\left(P\left(W_{L}\right)\right)^{\ell}\left(\left(P\left(W_{L}\right)\right)^{\ell}+\left(P\left(V_{L}\right)\right)^{\ell}\right)^{-1}$. In particular, if $\lim _{L \rightarrow \infty} \rho_{L}=\rho$, then $\Sigma_{N_{L}}^{V_{L}, W_{L}}$ converges in total variation as $L \rightarrow \infty$ to the geometric distribution with the parameter $\rho$. Furthermore, let $V_{L}=A_{n_{L}}^{\xi}=\left[\xi_{0}, \ldots, \xi_{n_{L}-1}\right] \in \mathcal{C}_{n_{L}}$ and $W_{L}=A_{m_{L}}^{\eta}=\left[\eta_{0}, \ldots, \eta_{m_{L}-1}\right] \in \mathcal{C}_{m_{L}}$ with $n_{L}, m_{L} \rightarrow \infty$ as $L \rightarrow \infty$ and suppose that $\xi, \eta$ are not periodic and not shifts of each other. Then

$$
\kappa_{A_{n_{L}}^{\xi}, A_{m_{L}}^{\eta}} \rightarrow \infty \text { as } L \rightarrow \infty
$$

and if also

$$
n_{L} \wedge m_{L}+\kappa_{A_{n_{L}}^{\xi}, A_{m_{L}}^{\eta}}-n_{L} \vee m_{L} \rightarrow \infty \text { as } L \rightarrow \infty
$$

then (2.17) holds true. In fact, (2.23) is satisfied for $P \times P$-almost all $(\xi, \eta) \in \Omega \times \Omega$ provided

$$
2 n_{L} \wedge m_{L}-n_{L} \vee m_{L}-3 v \ln \left(n_{L} \wedge m_{L}\right) \rightarrow \infty \text { as } L \rightarrow \infty
$$

where $v$ is from (2.12).

2.10. Remark. Recall that a sequence of random variables $\xi_{0}, \xi_{1}, \ldots$ is called $\phi$ mixing if

$$
\begin{gathered}
\phi(n)=\sup _{m \geq 0}\left\{\left|\frac{P(\Gamma \cap \Delta)}{P(\Gamma)}-P(\Delta)\right|:\right. \\
\left.P(\Gamma) \neq 0, \Gamma \in \mathcal{F}_{0, m}, \Delta \in \mathcal{F}_{m+n, \infty}\right\} \rightarrow \infty \text { as } n \rightarrow \infty
\end{gathered}
$$

where $\mathcal{F}_{k l}=\sigma\left\{\xi_{k}, \ldots, \xi_{l}\right\}$. It turns out that even when $\ell=1$ (conventional setup) and $q_{1, N}(n)=n$, in general, $\phi$-mixing does not suffice for Theorems 2.2 and 2.4 and Corollaries 2.3 and 2.5 to hold true. Indeed, consider an i.i.d. sequence $\eta_{0}, \eta_{1}, \ldots$ and set $\xi_{2 n}=\xi_{2 n+1}=\eta_{n}, n=0,1, \ldots$. Then $\xi_{0}, \xi_{1}, \ldots$ is a $\phi$-mixing identically distributed sequence but, as it is easy to see, the corresponding sums in Corollaries 2.3 and 2.5 will converge in distribution to random variables taking on only even integer values, and so they cannot be Poisson or geometric distributed. Unlike the case of $\phi$-mixing identically distributed sequences of random variables discussed above, in the shifts setup Theorems 2.6, 2.8 and Corollaries 2.7, 2.9 can be derived assuming only $\phi$-mixing when $\ell=1$ by using the technique from [16]. 


\section{Counting Arguments}

Let $\mathcal{N}_{N}$ be the set of $n \in\{1, \ldots, N\}$ such that all $q_{i, N}(n), i=1, \ldots, \ell$ are distinct and set $\hat{\mathcal{N}}_{N}=\{1, \ldots, N\} \backslash \mathcal{N}_{N}$, i.e. $\hat{\mathcal{N}}_{N}=\left\{n \in\{1, \ldots, N\}: q_{i, N}(n)=q_{j, N}(n)\right.$ for some $i, j=1, \ldots, \ell, i \neq j\}$. By Assumption 2.1(i),

$$
\# \hat{\mathcal{N}}_{N} \leq \frac{1}{2} K \ell(\ell-1)
$$

where $\# \Gamma$ denotes the cardinality of a set $\Gamma$.

Introduce also

$U_{N, M}=\left\{n \in\{1, \ldots, N\}:\left|q_{i, N}(n)-q_{j, N}(n)\right| \geq M\right.$ for all $\left.i, j=1, \ldots, \ell, i \neq j\right\}$.

By Assumption 2.1(i) for each pair $i \neq j$ and any $k$ there exist no more than $K$ nonnegative integers $n$ such that $q_{i, N}(n)-q_{j, N}(n)=k$, and so

$$
\#\left(\{1, \ldots, N\} \backslash U_{N, M}\right) \leq K M \ell(\ell-1) .
$$

We will need also the following semi-metrics between positive integers $k, l>0$,

$$
\delta_{N}(k, l)=\min _{1 \leq i, j \leq \ell}\left|q_{i, N}(k)-q_{j, N}(l)\right| .
$$

It follows from Assumption 2.1(i) that for any integers $n \in\{1, \ldots, N\}$ and $k \geq 0$,

$$
\#\left\{m: \delta_{N}(n, m)=k\right\} \leq K \ell(\ell-1) .
$$

For any integers $M, R \geq 1$ and $1 \leq n \leq N$ introduce the sets

$$
B_{n, N}^{M, R}=\left\{l: 1 \leq l \leq M, \delta_{N}(l, n)<R\right\} \quad \text { and } \quad B_{n, N}^{R}=B_{n, N}^{N, R} .
$$

By (3.3), for any $n$,

$$
\# B_{n, N}^{M, R} \leq \min (M, K R \ell(\ell-1))
$$

Next, set

$$
\sigma_{N}(n, m)=\max _{1 \leq j \leq \ell} \min _{1 \leq i \leq \ell}\left|q_{i, N}(n)-q_{j, N}(m)\right| .
$$

Then

$$
\#\left\{(m, n), m \neq n: \sigma_{N}(n, m)=0 \text { and either } n \in U_{N, 1} \text { or } m \in U_{N, 1}\right\} \leq K .
$$

Indeed, if either $n \in U_{N, 1}$ or $m \in U_{N, 1}$ and $\sigma_{N}(n, m)=0$ then, in fact, both $n \in U_{N, 1}$ and $m \in U_{N, 1}$. In order to see this, suppose, for instance, that $n \in$ $U_{N, 1}$ and $\sigma_{N}(n, m)=0$. Then there exist permutations $\eta$ and $\zeta$ of $\{1, \ldots, \ell\}$ such that $q_{\eta(1), N}(n)<q_{\eta(2), N}(n)<\ldots<q_{\eta(\ell), N}(n)$ and $q_{\eta(i), N}(n)=q_{\zeta(i), N}(m)$ for all $i=1, \ldots, \ell$, and so $q_{\zeta(1), N}(m)<q_{\zeta(2), N}(m)<\ldots<q_{\zeta(\ell), N}(m)$. The proof is the same assuming that $m \in U_{N, 1}$. Hence, $q_{i, N}(n)=q_{\eta^{-1} \zeta(i), N}(m), i=1, \ldots, \ell$. By Assumption 2.1(ii) there exists no more than $K$ pairs $m \neq n$ solving the latter system of equations, and so (3.5) follows.

\section{Poisson Distribution Limits FOR $\psi$-MiXing PROCESSES}

Set $p_{n, N}=P\left\{X_{n, N}=1\right\}=E X_{n, N}$ and $p_{n, l, N}=P\left\{X_{n, N}=1\right.$ and $\left.X_{l, N}=1\right\}=$ $E\left(X_{n, N} X_{l, N}\right)$ where $X_{n, N}, n=1, \ldots, N$ are the same as in Theorem 2.2. Then by Theorem 1 in [3] (warning the reader that the estimates there have an extra factor 2 due to a difference in the definition of $d_{T V}$ ),

$$
d_{T V}\left(\mathcal{L}\left(S_{N}\right), \operatorname{Pois}\left(\lambda_{N}\right)\right) \leq b_{1}+b_{2}+b_{3}
$$


where

$$
b_{1}=\sum_{n=1}^{N} \sum_{l \in B_{n, N}^{R}} p_{n, N} p_{l, N}, \quad b_{2}=\sum_{n=1}^{N} \sum_{n \neq l \in B_{n, N}^{R}} p_{n, l, N}
$$

and

$$
b_{3}=\sum_{n=1}^{N} s_{n, N} \text { with } s_{n, N}=E\left|E\left(X_{n, N}-p_{n, N} \mid \sigma\left\{X_{l, N}: l \in\{1, \ldots, N\} \backslash B_{n, N}^{R}\right\}\right)\right| .
$$

By Lemma 3.2 in [14, for each $n \in U_{N, M}$,

$$
p_{n, N}=P\left\{\xi_{q_{i, N}(n)} \in \Gamma \text { for } i=1, \ldots, \ell\right\} \leq(1+\psi(M))^{\ell}(\Phi(\Gamma))^{\ell}
$$

and for any $n$, clearly,

$$
p_{n, N} \leq P\left\{\xi_{q_{1, N}(n)} \in \Gamma\right\}=\Phi(\Gamma) .
$$

Hence, by (3.2), (3.4), (4.2), (4.4) and (4.5),

$$
\begin{aligned}
b_{1} \leq & N K R \ell^{2}(1+\Phi(M))^{2 \ell}(\Phi(\Gamma))^{2 \ell}+K^{2} M^{2} \ell^{4}(\Phi(\Gamma))^{2} \\
& +K M \ell^{2}\left(N+K R \ell^{2}\right)(1+\psi(M))^{2 \ell}(\Phi(\Gamma))^{\ell+1} .
\end{aligned}
$$

Next, if $\delta_{N}(n, l)=k \geq 1$ and $n, l \in U_{N, M}$ then by Lemma 3.2 in [14,

$$
\begin{gathered}
p_{n, l, N}=P\left\{\xi_{q_{i, N}(n)} \in \Gamma \text { and } \xi_{q_{i, N}(l)} \in \Gamma \text { for } i=1, \ldots \ell\right\} \\
\leq(1+\psi(M \wedge k))^{2 \ell}(\Phi(\gamma))^{2 \ell} .
\end{gathered}
$$

If $\delta_{N}(n, l)=k \geq 1$ and either $n \in U_{N, M}$ or $l \in U_{N, M}$ relying on Lemma 3.2 in [14] we see that

$$
p_{n, l, N} \leq(1+\psi(1))^{\ell+1}(\Phi(\Gamma))^{\ell+1}
$$

since if, for instance, $n \in U_{N, M}$ then we have $\left|q_{i, N}(n)-q_{j, N}(n)\right|>M$ for all $i \neq j$ and, in addition, $\left|q_{1, N}(l)-q_{j, N}(n)\right|=k \geq 1$ which yields (4.8). If $\delta_{N}(n, l)=k \geq 1$ and $n, l \notin U_{N, M}$ then $\left|q_{i, N}(n)-q_{j, N}(l)\right|=k \geq 1$ for some $i$ and $j$, and so in this case

$$
p_{n, l, N} \leq(1+\psi(k))^{2}(\Phi(\Gamma))^{2} .
$$

Next, suppose that $\delta_{N}(n, l)=0$ and either $n \in U_{N, M}$ or $l \in U_{N, M}$. then

$$
p_{n, l, N} \leq \min \left(p_{n, N}, p_{l, N}\right) \leq(1+\psi(M))^{\ell}(\Phi(\Gamma))^{\ell} .
$$

By (3.5) there exist no more than $K$ pairs $n \neq l, 1 \leq n, l \leq N$ such that $\sigma_{N}(n, l)=0$ and either $n \in U_{N, M}$ or $l \in U_{N, M}$ in which case we will rely on the estimate (4.10). If, on the other hand, $\sigma_{N}(n, l) \geq 1$ and, say, $n \in U_{N, M}$ then $\left|q_{i, N}(n)-q_{j, N}(n)\right| \geq$ $M \geq 1$ for all $i \neq j$ and $\left|q_{i, N}(n)-q_{m, N}(l)\right| \geq 1$ for all $i=1, \ldots, \ell$ and some $1 \leq m \leq \ell$. Similarly, if $\sigma_{N}(l, n) \geq 1$ and $l \in U_{N, M}$ then $\left|q_{i, N}(l)-q_{j, N}(l)\right| \geq M \geq 1$ for all $i \neq j$ and $\left|q_{i, N}(l)-q_{m, N}(n)\right| \geq 1$ for all $i=1, \ldots, \ell$ and some $1 \leq m \leq \ell$. In both cases we obtain the estimate (4.8) in view of Lemma 3.2 from [14].

Finally, if $\delta_{N}(n, l)=0$ and $n, l \notin U_{N, M}$ then by (4.5),

$$
p_{n, l, N} \leq p_{n, N} \leq \Phi(\Gamma) .
$$


It follows from (3.2)-(3.4) and (4.7)-4.11) that

$$
\begin{gathered}
b_{2} \leq N K R \ell^{2}(1+\psi(1))^{2 \ell}(\Phi(\Gamma))^{2 \ell}+K^{2} M^{2} \ell^{4} \Phi(\Gamma) \\
+K M \ell^{2}\left(N+K R \ell^{2}\right)(1+\psi(1))^{\ell+1}(\Phi(\Gamma))^{\ell+1} \\
+K^{2} M^{2} \ell^{4}(1+\psi(1))^{2}(\Phi(\Gamma))^{2}+2 K^{2} M R \ell^{4}(1+\psi(M))^{\ell}(\Phi(\Gamma))^{\ell} .
\end{gathered}
$$

Next, we claim that for any $n=1, \ldots, N$,

$$
\begin{aligned}
s_{n, N} \leq & 2^{2(\ell+2)}\left(2-(1+\psi(R))^{\ell+1}\right)^{-2} \psi(R) E\left|X_{n, \alpha}-p_{n, \alpha}\right| \\
& \leq 2^{2 \ell+5}\left(2-(1+\psi(R))^{\ell+1}\right)^{-2} \psi(R) p_{n, N} .
\end{aligned}
$$

Indeed, let $\mathcal{G}$ be the $\sigma$-algebra generated by all $\xi_{q_{i, N}(l)}, i=1, \ldots, \ell$ with $l \in$ $\{1, \ldots, N\} \backslash B_{n, N}^{R}$ and $\mathcal{H}$ be the $\sigma$-algebra generated by $\xi_{q_{i, N}(n)}, i=1, \ldots, \ell$. Since $\delta_{N}(n, l) \geq R$ for such $l$ and $n$ we derive from Lemma 3.3 in [14 that

$$
\psi(\mathcal{G}, \mathcal{H}) \leq 2^{2(\ell+2)} \psi(R)\left(2-(1+\psi(R))^{\ell+1}\right)^{-2}
$$

provided $\psi(R)<2^{\frac{1}{\ell+1}}-1$ which we assume. Since $\sigma\left\{X_{l}: l \in\{1, \ldots, N\} \backslash B_{n, N}^{R}\right\} \subset \mathcal{G}$ and $\sigma\left\{X_{n}\right\} \subset \mathcal{H}$ we obtain (4.13) from (2.1) and (4.14). Now by (3.2), (4.3)-(4.5) and (4.13),

$(4.15)$

$b_{3} \leq 2^{2 \ell+5}\left(2-(1+\psi(R))^{\ell+1}\right)^{-2} \psi(R)\left(K M \ell(\ell-1) \Phi(\Gamma)+N(1+\psi(M))^{\ell}(\Phi(\Gamma))^{\ell}\right)$.

Finally, (4.1), (4.6) (4.12) and (4.15) yield (2.4).

When (2.5) holds true for $\Gamma=\Gamma_{N}$ we can choose $M=M_{N} \rightarrow \infty$ and $R=$ $R_{N} \rightarrow \infty$ as $N \rightarrow \infty$ so that $M_{N}^{2} \Phi\left(\Gamma_{N}\right) \rightarrow 0$ and $M_{N} R_{N}\left(\Phi\left(\Gamma_{N}\right)\right)^{\ell} \rightarrow 0$ as $N \rightarrow \infty$ which in view of (2.4) will yield (2.6) proving Corollary 2.3 ,

\section{Geometric Distribution limits For $\psi$-MiXing PROCESSES}

Set for convenience $\Gamma_{0}=\Gamma, \Gamma_{1}=\Delta, X_{n, \alpha}=\prod_{i=1}^{\ell} \mathbb{I}_{\Gamma_{\alpha}}\left(\xi_{q_{i, N}(n)}\right), \alpha=0,1$ and $S_{L}=\sum_{n=1}^{L} X_{n, 0}$. Let $X_{n, \alpha}^{\prime}, n=1,2, \ldots, \alpha=0,1$ be a sequence of independent random variables such that $X_{n, \alpha}^{\prime}$ has the same distribution as $X_{n, \alpha}$. Set $\tau_{N}=$ $\min (\tau, N), S_{L}^{\prime}=\sum_{n=1}^{L} X_{n, 0}^{\prime}, \tau^{\prime}=\min \left\{n \geq 1: X_{n, 1}^{\prime}=1\right\}$ and $\tau_{N}^{\prime}=\min \left(\tau^{\prime}, N\right)$. Next, let $Y_{n, 0}$ and $Y_{n, 1}, n=1,2, \ldots$ be two independent of each other sequences of i.i.d. random variables such that

$$
P\left\{Y_{n, \alpha}=1\right\}=\Phi\left(\Gamma_{\alpha}\right)^{\ell}=1-P\left\{Y_{n, \alpha}=0\right\}, \alpha=0,1 .
$$

We can and will assume that all above random variables are defined on the same (sufficiently large) probability space. Set also

$$
S_{L}^{*}=\sum_{n=1}^{L} Y_{n, 0}, \tau^{*}=\min \left\{n \geq 0: Y_{n, 1}=1\right\} \text { and } \tau_{N}^{*}=\min \left(\tau^{*}, N\right) .
$$

Now observe that $S_{\tau^{*}}^{*}$ has by Lemma 3.1 from [15] the geometric distribution with the parameter

$$
\varrho=\frac{\Phi\left(\Gamma_{1}\right)^{\ell}}{\Phi\left(\Gamma_{1}\right)^{\ell}+\Phi\left(\Gamma_{0}\right)^{\ell}\left(1-\Phi\left(\Gamma_{1}\right)^{\ell}\right)}>\rho
$$

Next, we can write

$$
d_{T V}\left(\mathcal{L}\left(S_{\tau}\right), \operatorname{Geo}(\rho)\right) \leq A_{1}+A_{2}+A_{3}+A_{4}+A_{5}
$$


where $\quad A_{1}=d_{T V}\left(\mathcal{L}\left(S_{\tau}\right), \mathcal{L}\left(S_{\tau_{N}}\right)\right), \quad A_{2}=d_{T V}\left(\mathcal{L}\left(S_{\tau_{N}}\right), \mathcal{L}\left(S_{\tau_{N}^{\prime}}^{\prime}\right)\right)$, $A_{3}=d_{T V}\left(\mathcal{L}\left(S_{\tau_{N}^{\prime}}^{\prime}\right), \mathcal{L}\left(S_{\tau_{N}^{*}}^{*}\right)\right), \quad A_{4}=d_{T V}\left(\mathcal{L}\left(S_{\tau_{N}^{*}}^{*}\right), \mathcal{L}\left(S_{\tau^{*}}^{*}\right)\right) \quad$ and $\quad A_{5}=$ $d_{T V}(\operatorname{Geo}(\varrho), \operatorname{Geo}(\rho))$.

Introduce random vectors $\mathbf{X}_{N, \alpha}=\left\{X_{n, \alpha}, 0 \leq n \leq N\right\}, \alpha=0,1, \mathbf{X}_{N}=$ $\left\{\mathbf{X}_{N, 0}, \mathbf{X}_{N, 1}\right\}, \mathbf{X}_{N, \alpha}^{\prime}=\left\{X_{n, \alpha}^{\prime}, 0 \leq n \leq N\right\}, \alpha=0,1, \mathbf{X}_{N}^{\prime}=\left\{\mathbf{X}_{N, 0}^{\prime}, \mathbf{X}_{N, 1}^{\prime}\right\}$, $\mathbf{Y}_{N, \alpha}=\left\{Y_{n, \alpha}, 0 \leq n \leq N\right\}, \alpha=0,1$ and $\mathbf{Y}_{N}=\left\{\mathbf{Y}_{N, 0}, \mathbf{Y}_{N, 1}\right\}$. Observe that the event $\left\{S_{\tau} \neq S_{\tau_{N}}\right\}$ can occur only if $\tau>N$. Also, we can write $\{\tau>N\}=$ $\left\{X_{n, 0}=0\right.$ for all $\left.n=0,1, \ldots, N\right\}$ and $\left\{\tau^{\prime}>N\right\}=\left\{X_{n, 0}^{\prime}=0\right.$ for all $\left.n=0,1, \ldots, N\right\}$ Hence,

$$
\begin{gathered}
A_{1} \leq P\{\tau>N\}=P\left\{\tau^{\prime}>N\right\}+\mid P\left\{X_{n, 1}=0 \text { for } n=0,1, \ldots, N\right\} \\
-P\left\{X_{n, 1}^{\prime}=0 \text { for } n=0,1, \ldots, N\right\} \mid \leq P\left\{\tau^{\prime}>N\right\}+d_{T V}\left(\mathcal{L}\left(\mathbf{X}_{N, 1}\right), \mathcal{L}\left(\mathbf{X}_{N, 1}^{\prime}\right)\right)
\end{gathered}
$$

and similarly,

$$
P\left\{\tau^{\prime}>N\right\} \leq P\left\{\tau^{*}>N\right\}+d_{T V}\left(\mathcal{L}\left(\mathbf{X}_{N, 1}^{\prime}\right), \mathcal{L}\left(\mathbf{Y}_{N, 1}\right)\right) .
$$

Since $Y_{n, 1}, n=0,1, \ldots$ are i.i.d. random variables we obtain that

$$
P\left\{\tau^{*}>N\right\}=\left(P\left\{Y_{0,1}=0\right\}\right)^{N}=\left(1-\left(\Phi\left(\Gamma_{1}\right)\right)^{\ell}\right)^{N} .
$$

Next, we claim that

$$
\begin{gathered}
d_{T V}\left(\mathcal{L}\left(\mathbf{X}_{N, 1}^{\prime}\right), \mathcal{L}\left(\mathbf{Y}_{N, 1}\right)\right) \leq d_{T V}\left(\mathcal{L}\left(\mathbf{X}_{N}^{\prime}\right), \mathcal{L}\left(\mathbf{Y}_{N}\right)\right) \\
\leq \sum_{0 \leq n \leq N, \alpha=0,1} d_{T V}\left(\mathcal{L}\left(X_{n, \alpha}^{\prime}\right), \mathcal{L}\left(Y_{n, \alpha}\right)\right) .
\end{gathered}
$$

The first inequality above is clear and the second one holds true since for any Borel probability measures $\mu_{1}, \mu_{2}$ and $\tilde{\mu}_{1}, \tilde{\mu}_{2}$ on Borel measurable spaces $\mathcal{X}$ and $\tilde{\mathcal{X}}$, respectively, (see, for instance, [15]),

$$
d_{T V}\left(\mu_{1} \times \tilde{\mu}_{1}, \mu_{2} \times \tilde{\mu}_{2}\right) \leq d_{T V}\left(\mu_{1}, \mu_{2}\right)+d_{T V}\left(\tilde{\mu}_{1}, \tilde{\mu}_{2}\right) .
$$

Now, for any $n \in U_{N, M}$ and $\alpha=0,1$,

$$
d_{T V}\left(\mathcal{L}\left(X_{n, \alpha}^{\prime}, \mathcal{L}\left(Y_{n, \alpha}\right)\right)=\left|P\left\{X_{n, \alpha}^{\prime}=1\right\}-P\left\{Y_{n, \alpha}=1\right\}\right|\right.
$$

$=\mid P\left\{\xi_{q_{i, N}(n)} \in \Gamma_{\alpha}\right.$ for $\left.i=1, \ldots, \ell\right\}-\left(\Phi\left(\Gamma_{\alpha}\right)\right)^{\ell} \mid \leq\left((1+\psi(M))^{\ell}-1\right)\left(\Phi\left(\Gamma_{\alpha}\right)\right)^{\ell}$

where the last inequality follows from Lemma 3.2 in [14. By (3.2), (4.5) and (5.8) for any positive integer $N$ we can write

$$
\begin{gathered}
\left.d_{T V}\left(\mathcal{L}\left(\mathbf{X}_{N}^{\prime}\right), \mathcal{L}\left(\mathbf{Y}_{N}\right)\right) \leq\left(\left(\Phi\left(\Gamma_{0}\right)\right)^{\ell}+\left(\Phi\left(\Gamma_{1}\right)\right)^{\ell}\right)\right) N\left((1+\psi(M))^{\ell}-1\right) \\
+K M \ell(\ell-1)\left(\Phi\left(\Gamma_{0}\right)+\Phi\left(\Gamma_{1}\right)\right)
\end{gathered}
$$

Observe that

$$
d_{T V}\left(\mathcal{L}\left(\mathbf{X}_{N, 0}\right), \mathcal{L}\left(\mathbf{X}_{N, 0}^{\prime}\right)\right) \leq d_{T V}\left(\mathcal{L}\left(\mathbf{X}_{N}\right), \mathcal{L}\left(\mathbf{X}_{N}^{\prime}\right)\right) \text { and } A_{2} \leq d_{T V}\left(\mathcal{L}\left(\mathbf{X}_{N}\right), \mathcal{L}\left(\mathbf{X}_{N}^{\prime}\right)\right) .
$$

The first inequality in (5.10) is clear and the second one follows from the fact that $S_{\tau_{N}}=f\left(\mathbf{X}_{N}\right)$ and $S_{\tau_{N}^{\prime}}^{\prime}=f\left(\mathbf{X}_{N}^{\prime}\right)$ for a certain function $f:\{0,1\}^{2 L} \rightarrow\{1, \ldots, N\}$. We will estimate next $d_{T V}\left(\mathcal{L}\left(\mathbf{X}_{N}\right), \mathcal{L}\left(\mathbf{X}_{N}^{\prime}\right)\right)$ relying on [3].

By Theorem 3 in $[3$,

$$
d_{T V}\left(\mathcal{L}\left(\mathbf{X}_{N}\right), \mathcal{L}\left(\mathbf{X}_{N}^{\prime}\right)\right) \leq 2 b_{1}+2 b_{2}+b_{3}+2 \sum_{0 \leq n \leq N, \alpha=0,1} p_{n, \alpha}^{2}
$$


where for $\alpha=0,1$ and $n \in U_{N, M}$,

$$
p_{n, \alpha}=P\left\{X_{n, \alpha}=1\right\}=P\left\{\xi_{q_{i, N}(n)} \in \Gamma_{\alpha} \text { for } i=1, \ldots, \ell\right\} \leq(1+\psi(M))^{\ell}\left(\Phi\left(\Gamma_{\alpha}\right)\right)^{\ell}
$$

with the latter inequality satisfied by Lemma 3.2 in [14]. In order to define $b_{1}, b_{2}$ and $b_{3}$ we introduce the set

$$
B_{n}^{N, R}=B_{n, N}^{N, R}=\left\{(l, 0),(l, 1): 0 \leq l \leq N, \delta_{N}(l, n) \leq R\right\}
$$

where an integer $R>0$ is another parameter. Set also $I_{N}=\{(n, \alpha): 0 \leq n \leq$ $N, \alpha=0,1\}$. Then

$$
\begin{gathered}
b_{1}=\sum_{(n, \alpha) \in I_{N}} \sum_{(l, \beta) \in B_{n}^{N, R}} p_{n, \alpha} p_{l, \beta}, \\
b_{2}=\sum_{(n, \alpha) \in I_{N}} \sum_{(n, \alpha) \neq(l, \beta) \in B_{n}^{N, R}} p_{(n, \alpha),(l, \beta)},
\end{gathered}
$$

where $p_{(n, \alpha),(l, \beta)}=E\left(X_{n, \alpha} X_{l, \beta}\right)$, and

$$
b_{3}=\sum_{(n, \alpha) \in I_{N}} s_{n, \alpha}
$$

where

$$
s_{n, \alpha}=E\left|E\left(X_{n, \alpha}-p_{n, \alpha} \mid \sigma\left\{X_{l, \beta}:(l, \beta) \in I_{N} \backslash B_{n}^{N, R}\right\}\right)\right| .
$$

By Assumption 2.1, for any $i, j, n$ and $k$ there exists at most $K$ of $l$ 's such that $q_{i, N}(n)-q_{j, N}(l)=k$. It follows from here that

$$
\# B_{n}^{N, R} \leq K \ell^{2} R .
$$

It follows from (3.2), (5.12), (5.13) and (5.16), similarly to (4.6), that

$$
\begin{gathered}
b_{1} \leq 2 N \ell^{2} R(1+\psi(1))^{2 \ell}\left(\left(\Phi\left(\Gamma_{0}\right)\right)^{2 \ell}+\left(\Phi\left(\Gamma_{1}\right)\right)^{2 \ell}\right) \\
+K \ell^{2}(1+\psi(1))^{\ell+1}(N+R)\left(\left(\Phi\left(\Gamma_{0}\right)\right)^{\ell+1}+\left(\Phi\left(\Gamma_{1}\right)\right)^{\ell+1}\right) \\
+K^{2} \ell^{4}(1+\psi(1))^{2}\left(\left(\Phi\left(\Gamma_{0}\right)\right)^{2}+\left(\Phi\left(\Gamma_{1}\right)\right)^{2}\right) .
\end{gathered}
$$

Since $\Gamma_{0} \cap \Gamma_{1}=\emptyset$,

$$
\begin{aligned}
p_{(n, \alpha),(l, \beta)}= & P\left\{X_{n, \alpha}=X_{l, \beta}=1\right\}=0 \text { if } n=l, \beta=1-\alpha \\
& \text { and always } p_{(n, \alpha),(l, \beta)} \leq p_{(l, \beta)} .
\end{aligned}
$$

Similarly to Section 4 we estimate $p_{(n, \alpha),(l, \beta)}$ by the right hand sides of (4.7)-(4.11) in the corresponding cases replacing $\Phi(\Gamma)$ there by $\Phi\left(\Gamma_{0}\right)+\Phi\left(\Gamma_{1}\right)$ here, namely, if $\delta_{N}(n, l)=k \geq 1$ and $n, l \in U_{N, M}$ we estimate it via the right hand side of (4.7), if either $n \in U_{N, M}$ or $l \in U_{N, M}$ and either $\delta_{N}(n, l)=k \geq 1$ or $\delta_{N}(n, l)=0$ and $\sigma_{N}(n, l) \geq 1$ we estimate it via the right hand side of (4.8), if $\delta_{N}(n, l)=k \geq 1$ and $n, l \notin U_{N, M}$ we estimate it via the right hand side of (4.9), if $\sigma_{N}(n, l)=0$ and either $n \in U_{N, M}$ or $l \in U_{N, M}$ we estimate it by the right hand side of (4.10) and, finally, if $\sigma_{N}(n, l)=0$ and $n, l \notin U_{N, M}$ we estimate it via the right hand side of 
(4.11). These estimates together with counting estimates of Section 3 yield

$$
\begin{gathered}
b_{2} \leq 2 N K R \ell^{2}(1+\psi(1))^{2 \ell}\left(\Phi\left(\Gamma_{0}\right)+\Phi\left(\Gamma_{1}\right)\right)^{2 \ell} \\
+2 K M \ell^{2}\left(N+K R \ell^{2}\right)(1+\psi(1))^{\ell+1}\left(\Phi\left(\Gamma_{0}\right)+\Phi\left(\Gamma_{1}\right)\right)^{\ell+1} \\
+K^{2} M^{2} \ell^{4}\left(\Phi\left(\Gamma_{0}\right)+\Phi\left(\Gamma_{1}\right)\right)+K^{2} M^{2} \ell^{4}(1+\psi(1))^{2}\left(\Phi\left(\Gamma_{0}\right)+\Phi\left(\Gamma_{1}\right)\right)^{2} \\
+2 K^{2} M R \ell^{4}(1+\psi(M))^{\ell}\left(\Phi\left(\Gamma_{0}\right)+\Phi\left(\Gamma_{1}\right)\right)^{\ell} .
\end{gathered}
$$

In the same way as in Section 4 we obtain that

$$
\begin{gathered}
s_{n, \alpha} \leq 2^{2(\ell+2)}\left(2-(1+\psi(R))^{\ell+1}\right)^{-2} \psi(R) E\left|X_{n, \alpha}-p_{n, \alpha}\right| \\
\leq 2^{2 \ell+5}\left(2-(1+\psi(R))^{\ell+1}\right)^{-2} \psi(R) p_{n, \alpha}
\end{gathered}
$$

where $s_{n, \alpha}$ is the same as in (5.15). Hence, by (5.12), (5.15) and (5.21),

$$
\left.+N(1+\psi(M))^{\ell}\left(\left(\Phi\left(\Gamma_{0}\right)\right)^{\ell}+\left(\Phi\left(\Gamma_{1}\right)\right)^{\ell}\right)\right) .
$$

Next, in the same way as in the estimate of $A_{2}$ we conclude that

$$
A_{3} \leq d_{T V}\left(\mathcal{L}\left(\mathbf{X}_{N}^{\prime}\right), \mathcal{L}\left(\mathbf{Y}_{N}\right)\right)
$$

which together with (5.9) estimates $A_{3}$.

As in the estimate of $A_{1}$ we see that

$$
A_{4} \leq P\left\{\tau^{*}>N\right\} \leq\left(1-\left(\Phi\left(\Gamma_{1}\right)\right)^{\ell}\right)^{N}
$$

since $Y_{n, 0}, n=0,1, \ldots$ are i.i.d. random variables.

Since $\varrho>\rho$ we obtain

$$
\begin{gathered}
A_{5} \leq \sum_{k=0}^{\infty}\left|\varrho(1-\varrho)^{k}-\rho(1-\rho)^{k}\right| \leq 2 \sum_{k=1}^{\infty}\left((1-\rho)^{k}-(1-\varrho)^{k}\right) \\
=2(1-\rho) \rho^{-1}-2(1-\varrho) \varrho^{-1}=2 \frac{\rho-\rho}{\rho \varrho}=2\left(\Phi\left(\Gamma_{0}\right)\right)^{\ell} .
\end{gathered}
$$

Collecting (5.3)-(5.12), (5.17), (5.21), (5.20) and (5.21)-(5.25) we derive (2.8).

In order to prove Corollary 2.5 we rely on the estimate (2.8) with $\Gamma=\Gamma_{N}$ and $\Delta=\Delta_{N}$ choosing $M=M_{N} \rightarrow \infty$ and $R=R_{N} \rightarrow \infty$ as $N \rightarrow \infty$ so that

$$
\begin{gathered}
\lim _{N \rightarrow \infty} N\left(\Phi\left(\Delta_{N}\right)\right)^{\ell}=\infty \\
\lim _{N \rightarrow \infty}\left(\left(\Phi\left(\Gamma_{N}\right)\right)^{\ell}+\left(\Phi\left(\Delta_{N}\right)\right)^{\ell}\right) N \psi\left(M_{N}\right)=0 \\
\lim _{N \rightarrow \infty} N \psi\left(R_{N}\right)\left(\left(\Phi\left(\Gamma_{N}\right)\right)^{\ell}+\left(\Phi\left(\Delta_{N}\right)\right)^{\ell}\right)=0 \\
\lim _{N \rightarrow \infty} N\left(R_{N}+M_{N}\right)\left(\left(\Phi\left(\Gamma_{N}\right)\right)^{\ell+1}+\left(\Phi\left(\Delta_{N}\right)\right)^{\ell+1}\right)=0 \text { and } \\
\lim _{N \rightarrow \infty}\left(M_{N}^{2}\left(\Phi\left(\Gamma_{N}\right)+\Phi\left(\Delta_{N}\right)\right)+M_{N} R_{N}\left(\left(\Phi\left(\Gamma_{N}\right)\right)^{\ell}+\left(\Phi\left(\Delta_{N}\right)\right)^{\ell}\right)\right)=0 .
\end{gathered}
$$

which is clearly possible since $\psi(n) \rightarrow 0$ as $n \rightarrow \infty$. This together with (2.8) yields (2.10).

\section{Poisson Distribution Limits For Shifts}

Let $V \in \mathcal{F}_{0, n-1}$ and set $X_{k, N}=X_{k, N}^{V}=\prod_{i=1}^{\ell} \mathbb{I}_{V} \circ T^{q_{i, N}(k)}$. Then $S_{N}=S_{N}^{V}=$ $\sum_{k=1}^{N} X_{k, N}$. Set $p_{k, N}=P\left\{X_{k, N}=1\right\}$ and $p_{k, l, N}=P\left\{X_{k, N}=1\right.$ and $\left.X_{l, N}=1\right\}$. Then, again, by Theorem 1 from [3] we obtain

$$
d_{T V}\left(\mathcal{L}\left(S_{N}\right), \operatorname{Pois}\left(\lambda_{N}\right)\right) \leq b_{1}+b_{2}+b_{3}
$$

where $b_{1}, b_{2}$ and $b_{3}$ are defined by (4.2) and (4.3) though their estimates will proceed now somewhat differently than in Section 4 
Since $V \in \mathcal{F}_{0, n-1}$, it follows from Lemma 3.2 in [14 that for any $k \in U_{N, n}$,

$$
p_{k, N}=E X_{k, N} \leq(1+\psi(1))^{\ell}(P(V))^{\ell}
$$

while when $k \notin U_{N, n}$ we can always write

$$
p_{k, N} \leq E\left(\mathbb{I}_{V} \circ T^{q_{1, N}(k)}\right)=P(V) .
$$

Hence, by (3.2) and (3.4) we conclude that

$$
b_{1}=\sum_{k=1}^{N} \sum_{l \in B_{k, N}^{R}} p_{k, N} p_{l, N} \leq K^{2} R \ell^{4} P(V)+N K R \ell^{2}(P(V))^{\ell+1} .
$$

In order to estimate $p_{k, l, N}$ we observe that if $|i-j|<\pi(V)$ then $\left(\mathbb{I}_{V} \circ T^{i}\right)\left(\mathbb{I}_{V} \circ\right.$ $\left.T^{j}\right)=0$. Hence, $p_{k, l, N}=0$ if $\delta_{N}(k, l)<\pi(V)$. Now suppose that $\delta_{N}(k, l)=d$ with $\pi(V) \leq d<n$,

$$
q_{i_{1}, N}(k) \leq q_{i_{2}, N}(k) \leq \ldots \leq q_{i_{\ell}, N}(k) \text { and } q_{j_{1}, N}(l) \leq q_{j_{2}, N}(l) \leq \ldots \leq q_{j_{\ell}, N}(l) .
$$

Assume that the pair $k, l$ does not belong to the exceptional set $D_{N}$ of cardinality at most $K$ appearing in Assumption 2.1(iii). Since $\delta_{N}(k, l)=d \geq \pi(V)$, it follows that

$$
\text { either } q_{j_{1}, N}(l) \leq q_{i_{1}, N}(k)-d \text { or } q_{j_{\ell}, N}(l) \geq q_{i_{\ell}, N}(k)+d
$$

and in view of Assumption 2.1(iii) only one of these inequalities can hold true. Assume, for instance, that the first inequality in (6.5) holds true and let $r=$ $q_{i_{1}, N}(k)-q_{j_{1}, N}(l)$. Then $r \geq d \geq \pi(V)$. If $r \geq n$ then by Lemma 3.2 from [14] (essentially, by the definition of the $\psi$-mixing coefficient itself),

$$
p_{k, l, N}=E\left(X_{k, N} X_{l, N}\right) \leq E\left(X_{k, n} \mathbb{I}_{V} \circ T^{q_{j_{1}, N}(l)}\right) \leq(1+\psi(1)) p_{k, N} P(V) .
$$

Suppose that $\pi(V) \leq r<n$. Then the sets $Q_{0}=\left\{q_{j_{1}, N}(l), q_{j_{1}, N}(l)+\right.$ $\left.1, \ldots, q_{j_{1}, N}(l)+n-1\right\}$ and $Q_{1}=\left\{q_{i_{1}, N}(k)+n-r, q_{i_{1}, N}(k)+n-r+1, \ldots, q_{i_{1}, N}(k)+\right.$ $n-1\}$ are disjoint, and so it follows by Lemma 3.2 from [14 that in this case,

$$
\begin{gathered}
p_{k, l, N}=E\left(X_{k, N} X_{l, N}\right) \leq E\left(\mathbb{I}_{V} \circ T^{q_{j_{1}, N}(l)} \mathbb{I}_{V} \circ T^{q_{i_{1}, N}(k)}\right) \\
\leq E\left(\mathbb{I}_{V} \circ T^{q_{j_{1}, N}(l)} \mathbb{I}_{T^{n-r} V} \circ T^{q_{i_{1}, N}(k)+n-r} V\right) \leq(1+\psi(1)) P(V) P\left(T^{n-r} V\right)
\end{gathered}
$$

where we used that $V \subset T^{-a} T^{a} V$ for any integer $a \geq 0$. If, in addition, $k \in U_{N, n}$ then the sets $Q_{0}=\left\{q_{j_{1}, N}(l), q_{j_{1}, N}(l)+1, \ldots, q_{j_{1}, N}(l)+n-1\right\}, Q_{1}=\left\{q_{i_{1}, N}(k)+\right.$ $\left.n-r, q_{i_{1}, N}(k)+n-r+1, \ldots, q_{i_{1}, N}(k)+n-1\right\}$ and $Q_{m}=\left\{q_{i_{m}, N}(k), q_{i_{m}, N}(k)+\right.$ $\left.1, \ldots, q_{i_{m}, N}(k)+n-1\right\}, m=1, \ldots, \ell$ are disjoint and we obtain then from Lemma 3.2 in 14 that

$$
p_{k, l, N}=E\left(X_{k, N} X_{l, N}\right) \leq(1+\psi(1))^{\ell}(P(V))^{\ell} P\left(T^{n-r} V\right) .
$$

If the second inequality in (6.5) holds true then we obtain (6.6) if $r=$ $q_{j_{\ell}, N}(l)-q_{i_{\ell}, N}(k) \geq n$, while if $\pi(V) \leq r<n$ then we use that the sets $Q_{\ell}=$ $\left\{q_{i_{\ell}, N}(k), q_{i_{\ell}, N}(k)+1, \ldots, q_{i_{\ell}, N}(k)+n-1\right\}$ and $Q_{\ell+1}=\left\{q_{j_{\ell}, N}(l)+n-r, q_{j_{\ell}, N}(l)+n-\right.$ $\left.r+1, \ldots, q_{j_{\ell}, N}(l)+n-1\right\}$ are disjoint which yields (6.7) by Lemma 3.2 from [14]. If, in addition, $k \in U_{N, n}$ then all sets $Q_{m}=\left\{q_{i_{m}, N}(k), q_{i_{m}, N}(k)+1, \ldots, q_{i_{m}, N}(k)+n-1\right\}$, $m=1, \ldots, \ell$ and $Q_{\ell+1}=\left\{q_{j_{\ell}, N}(l)+n-r, q_{j_{\ell}, N}(l)+n-r+1, \ldots, q_{j_{\ell}, N}(l)+n-1\right.$ are disjoint, and so by Lemma 3.2 from [14] we obtain the estimate (6.8) again. Finally, suppose that $\delta_{N}(k, l)=d \geq n$. Then, applying Lemma 3.2 from [14] we 
see that the estimate (6.6) holds true again. Observe that by Assumption 2.1(i) for any $N \geq 1, i=1, \ldots, \ell$ and integers $k \geq 0$ and $r$,

$$
\#\left\{l \geq 0: q_{i, N}(k)-q_{j, N}(l)=r \text { for some } 1 \leq j \leq \ell\right\} \leq \ell K .
$$

Now, it follows from (3.2)-(3.4), (6.2), (6.3) and (6.6)-(6.9) that

$$
\begin{gathered}
(6.10) b_{2}=\sum_{k=1}^{N} \sum_{k \neq l \in B_{k, N}^{R}} p_{k, l, N}=\sum_{1 \leq k \leq N, k \notin U_{N, n}} \sum_{l: \pi(V) \leq \delta_{N}(k, l)<R} p_{k, l, N} \\
+\sum_{k \in U_{N, n}} \sum_{l: \pi(V) \leq \delta_{N}(k, l)<R} p_{k, l, N} \leq K^{2} \ell^{4}(1+\psi(1)) n R(P(V))^{2} \\
+K^{2} \ell^{3}(1+\psi(1)) n P(V) \sum_{r=\pi(V)}^{n-1} P\left(T^{n-r} V\right)+K \ell^{2}(1+\psi(1))^{\ell} N R(P(V))^{\ell+1} \\
+K \ell\left((1+\psi(1))^{\ell} N(P(V))^{\ell}\right) \sum_{r=\pi(V)}^{n-1} P\left(T^{n-r} V\right)=\left(K^{2} \ell^{3}(1+\psi(1)) n P(V)\right. \\
+K \ell\left((1+\psi(1))^{\ell} N(P(V))^{\ell}\right)\left(R \ell P(V)+\sum_{r=\pi(V)}^{n-1} P\left(T^{n-r} V\right)\right) .
\end{gathered}
$$

Next, we estimate $s_{k, N}$ and $b_{3}$ defined by (4.3). Let $\mathcal{G}=\mathcal{G}_{k}$ be the $\sigma$-algebra generated by the sets $T^{-q_{i, N}(l)} V, i=1, \ldots, \ell ; l \in\{1, \ldots, N\} \backslash B_{k, N}^{R}$ and $\mathcal{H}=\mathcal{H}_{k}$ be the $\sigma$-algebra generated by the sets $T^{-q_{i, N}(k)} V, i=1, \ldots, \ell$. Since $\delta_{N}(k, l) \geq R$ for $l \notin B_{k, N}^{R}$ and $V \in \mathcal{F}_{0, n-1}$, we derive from Lemma 3.3 in [14 that for $n<R<N$,

$$
\psi(\mathcal{G}, \mathcal{H}) \leq 2^{2(\ell+2)} \psi(R-n)\left(2-(1+\psi(R-n))^{\ell+1}\right)^{-2},
$$

and so

$$
\begin{gathered}
s_{k, N} \leq 2^{2(\ell+2)} \psi(R-n)\left(2-(1+\psi(R-n))^{\ell+1}\right)^{-2} E\left|X_{k, N}-p_{k, N}\right| \\
\leq 2^{2 \ell+5} \psi(R-n) p_{k, N}\left(2-(1+\psi(R-n))^{\ell+1}\right)^{-2} .
\end{gathered}
$$

Hence, by (3.2), (6.2), (6.3) and (6.12),

$$
\begin{gathered}
b_{3}=\sum_{k=1}^{N} s_{k, N} \leq 2^{2 \ell+5} \psi(R-n)\left(2-(1+\psi(R-n))^{\ell+1}\right)^{-2} \\
\times\left(K \ell^{2} n P(V)+(1+\psi(1))^{\ell} N(P(V))^{\ell}\right)
\end{gathered}
$$

Finally, collecting (6.1), (6.4), (6.9) and (6.13) we derive (2.13) completing the proof of Theorem 2.6.

Corollary 2.7 follows from the estimate (2.13) choosing $R=R_{L}=2 n_{L}$ and in view of (2.12) we obtain Corollary 2.7 for $V_{N_{L}}=A_{n_{L}}^{\eta}$ since

$$
\pi\left(A_{n}^{\eta}\right) \rightarrow \infty \text { as } n \rightarrow \infty
$$

when $\eta$ is a nonperiodic sequence. Indeed, $\pi\left(A_{n}^{\eta}\right)$ is, clearly, nondecreasing in $n$, and so $\lim _{n \rightarrow \infty} \pi\left(A_{n}^{\eta}\right)=r$ exists. If $r<\infty$ then there exists $n_{0} \geq 1$ such that $\pi\left(A_{n}^{\eta}\right)=r$ for all $n \geq n_{0}$ which means that $\eta$ is periodic with the period $r$, and so $r=\infty$ since $\eta$ is not periodic.

\section{Geometric Distribution Limits For Shifts}

It will be convenient to set $V^{(0)}=V \in \mathcal{F}_{0, n-1}, V^{(1)}=W \in \mathcal{F}_{0, m-1}$ and

$$
X_{k, N}^{(\alpha)}=\prod_{i=1}^{\ell} \mathbb{I}_{V^{(\alpha)}} \circ T^{q_{i, N}(k)}, \alpha=0,1
$$

so that

$$
\tau=\tau_{V^{(1)}}=\min \left\{k \geq 1: X_{k, N}^{(1)}=1\right\} \text { and } \Sigma_{N}^{V^{(0)}, V^{(1)}}=\sum_{k=1}^{\tau} X_{k, N}^{(0)} .
$$


Set also $S_{L}=\sum_{k=1}^{L} X_{k, N}^{(0)}$, so that $S_{\tau}=\Sigma_{N}^{V^{(0)}, V^{(1)}}$, and denote $\tau_{N}=\min (\tau, N)$. Let $\left\{Y_{k, N}^{(\alpha)}: k \geq 1, \alpha=0,1\right\}$ be a sequence of independent Bernoulli random variables such that $Y_{k, N}^{(\alpha)}$ has the same distribution as $X_{k, N}^{(\alpha)}$. Set

$$
S_{L}^{\prime}=\sum_{k=1}^{L} Y_{k, N}^{(0)}, \tau^{\prime}=\min \left\{k \geq 1: Y_{k, N}^{(1)}=1\right\} \text { and } \tau_{N}^{\prime}=\min \left(\tau^{\prime}, N\right) .
$$

Let now $\left\{Z_{k, N}^{(\alpha)}: k \geq 1\right\}, \alpha=0,1$ be two independent of each other sequences of i.i.d. Bernoulli random variables such that

$$
P\left\{Z_{k, N}^{(\alpha)}=1\right\}=\left(P\left(V^{\alpha)}\right)\right)^{\ell}=1-P\left\{Z_{k, N}^{(\alpha)}=0\right\}, \alpha=0,1 .
$$

We can and will assume that all above random variables are defined on the same (sufficiently large) probability space. Set also

$$
S_{L}^{*}=\sum_{k=1}^{L} Z_{k, N}^{(0)}, \tau^{\prime}=\min \left\{k \geq 1: Z_{k, N}^{(1)}=1\right\} \text { and } \tau_{N}^{*}=\min \left(\tau^{*}, N\right) .
$$

By Lemma 3.1 from [14 the sum $S_{\tau^{*}}^{*}$ has the geometric distribution with the parameter

$$
\varrho=\frac{\left(P\left(V^{(1)}\right)\right)^{\ell}}{\left(P\left(V^{(1)}\right)\right)^{\ell}+\left(P\left(V^{(0)}\right)\right)^{\ell}\left(1-\left(P\left(V^{(1)}\right)\right)^{\ell}\right)}>\rho
$$

where $\left.\rho=\left(P\left(V^{(1)}\right)\right)^{\ell}\left(P\left(V^{(1)}\right)\right)^{\ell}+\left(P\left(V^{(0)}\right)\right)^{\ell}\right)^{-1}$.

Next, we can write

$$
d_{T V}\left(\mathcal{L}\left(S_{\tau}\right), \operatorname{Geo}(\rho)\right) \leq A_{1}+A_{2}+A_{3}+A_{4}+A_{5}
$$

where $A_{1}=d_{T V}\left(\mathcal{L}\left(S_{\tau}\right), \mathcal{L}\left(S_{\tau_{N}}\right)\right), \quad A_{2}=d_{T V}\left(\mathcal{L}\left(S_{\tau_{N}}\right), \mathcal{L}\left(S_{\tau_{N}^{\prime}}^{\prime}\right)\right)$, $A_{3}=d_{T V}\left(\mathcal{L}\left(S_{\tau_{N}^{\prime}}^{\prime}\right), \mathcal{L}\left(S_{\tau_{N}^{*}}^{*}\right)\right), \quad A_{4}=d_{T V}\left(\mathcal{L}\left(S_{\tau_{N}^{*}}^{*}\right), \mathcal{L}\left(S_{\tau^{*}}^{*}\right)\right) \quad$ and $\quad A_{5}=$ $d_{T V}(\operatorname{Geo}(\varrho), \operatorname{Geo}(\rho))$.

Introduce random vectors $\mathbf{X}_{N}^{(\alpha)}=\left\{X_{k, n}^{(\alpha)}, 1 \leq k \leq N\right\}, \alpha=0,1, \mathbf{X}_{N}=$ $\left\{\mathbf{X}_{N}^{(0)}, \mathbf{X}_{N}^{(1)}\right\}, \mathbf{Y}_{N}^{(\alpha)}=\left\{Y_{n, N}^{(\alpha)}, 1 \leq k \leq N\right\}, \alpha=0,1, \mathbf{Y}_{N}=\left\{\mathbf{Y}_{N}^{(0)}, \mathbf{Y}_{N}^{(1)}\right\}$, $\mathbf{Y}_{N}^{(\alpha)}=\left\{Y_{n, N}^{(\alpha)}, 1 \leq k \leq N\right\}, \alpha=0,1$ and $\mathbf{Y}_{N}=\left\{\mathbf{Y}_{N}^{(0)}, \mathbf{Y}_{N}^{(1)}\right\}$. Observe that the event $\left\{S_{\tau} \neq S_{\tau_{N}}\right\}$ can occur only if $\tau>N$. Also, we can write $\{\tau>N\}=$ $\left\{X_{n, N}^{(1)}=0\right.$ for all $\left.k=1, \ldots, N\right\}$ and $\left\{\tau^{\prime}>N\right\}=\left\{Y_{n, 0}^{(1)}=0\right.$ for all $\left.k=1, \ldots, N\right\}$ Hence,

$$
\begin{aligned}
A_{1} \leq P & \{\tau>N\}=P\left\{\tau^{\prime}>N\right\}+\mid P\left\{X_{n, N}^{(1)}=0 \text { for } n=1, \ldots, N\right\} \\
& -P\left\{Y_{n, N}^{(1)}=0 \text { for } n=0,1, \ldots, N\right\} \mid \leq P\left\{\tau^{*}>N\right\} \\
& +d_{T V}\left(\mathcal{L}\left(\mathbf{Y}_{N}\right), \mathcal{L}\left(\mathbf{Z}_{N}\right)\right)+d_{T V}\left(\mathcal{L}\left(\mathbf{X}_{N}\right), \mathcal{L}\left(\mathbf{Y}_{N}\right)\right) .
\end{aligned}
$$

Since $Z_{k, N}^{(1)}, k=0,1, \ldots$ are i.i.d. random variables we obtain that

$$
P\left\{\tau^{*}>N\right\}=\left(1-\left(P\left(V^{(1)}\right)^{\ell}\right)\right)^{N} .
$$

It is also not difficult to understand (see p.p. 1534-1535 in [14]) that

$$
d_{T V}\left(\mathcal{L}\left(\mathbf{Y}_{N}\right), \mathcal{L}\left(\mathbf{Z}_{N}\right)\right) \leq \sum_{1 \leq k \leq N, \alpha=0,1} d_{T V}\left(\mathcal{L}\left(Y_{k, N}^{(\alpha)}\right), \mathcal{L}\left(Z_{k, N}^{(\alpha)}\right)\right) .
$$


If $k \in U_{N, n \vee m}$ then by (7.6) and Lemma 3.2 from [14] similarly to (5.9) we obtain that,

$$
\begin{gathered}
d_{T V}\left(\mathcal{L}\left(Y_{k, N}^{(\alpha)}\right), \mathcal{L}\left(Z_{k, N}^{(\alpha)}\right)\right)=\left|P\left\{Y_{k, N}^{(\alpha)}=1\right\}-P\left\{Z_{k, N}^{(\alpha)}=1\right\}\right| \\
=\left|P\left\{\cap_{i=1}^{\ell} T^{-q_{i, N}(k)} V^{(\alpha)}\right\}-\left(P\left(V^{(\alpha)}\right)\right)^{\ell}\right| \leq\left((1+\psi(n \vee m))^{\ell}-1\right)\left(P\left(V^{(\alpha)}\right)\right)^{\ell}
\end{gathered}
$$

It follows from (3.2), (77.6) and (7.7) that

$$
\begin{aligned}
& d_{T V}\left(\mathcal{L}\left(\mathbf{Y}_{N}, \mathcal{L}\left(\mathbf{Z}_{N}\right)\right) \leq K \ell^{2}(n \vee m)\left(P\left(V^{(0}\right)\right)+P\left(V^{(1)}\right)\right. \\
& \left.+N\left(\left(P\left(V^{(0}\right)\right)\right)^{\ell}+\left(P\left(V^{(1)}\right)\right)^{\ell}\right)\left((1+\psi(n \vee m))^{\ell}-1\right) .
\end{aligned}
$$

Next, we observe that by Theorem 3 in [3],

$$
A_{2} \leq d_{T V}\left(\mathcal{L}\left(\mathbf{X}_{N}\right), \mathcal{L}\left(\mathbf{Y}_{N}\right)\right) \leq 2 b_{1}+2 b_{2}+b_{3}+2 \sum_{1 \leq k \leq N, \alpha=0,1}\left(p_{k, N}^{(\alpha)}\right)^{2}
$$

where $p_{k, N}^{(\alpha)}=P\left\{X_{k, N}^{(\alpha)}=1\right\}$ and if $k \in U_{N, n \vee m}$ then (in the same way as in (6.2) by Lemma 3.2 in [14,

$$
p_{k, N}^{(\alpha)} \leq(1+\psi(n \vee m))^{\ell}\left(P\left(V^{(\alpha)}\right)\right)^{\ell},
$$

while the definitions of $b_{1}, b_{2}$ and $b_{3}$ are similar to Section 6 taking into account the additional parameter $\alpha$. Namely, setting

$$
B_{k}^{N, R}=\{(l, 0),(l, 1): 1 \leq l \leq N, \delta(k, l) \leq R\}, p_{k, l, N}^{\alpha, \beta}=E\left(X_{k, N}^{(\alpha)} X_{l, N}^{(\beta)}\right)
$$

and $I_{N}=\{(k, \alpha): 1 \leq k \leq N, \alpha=0,1\}$ we have

$$
\begin{gathered}
b_{1}=\sum_{(k, \alpha) \in I_{N}} \sum_{(l, \beta) \in B_{k}^{N, R}} p_{k, N}^{(\alpha)} p_{l, N}^{(\beta)}, \\
b_{2}=\sum_{(k, \alpha) \in I_{N}} \sum_{(k, \alpha) \neq(l, \beta) \in B_{k}^{N, R}} p_{k, l, N}^{(\alpha, \beta)} \text { and } \\
b_{3}=\sum_{(k, \alpha) \in I_{N}} s_{k, N}^{(\alpha)} \text { where } \\
s_{k, N}^{(\alpha)}=E\left|E\left(X_{k, N}^{(\alpha)}-p_{k, N}^{(\alpha)} \mid \sigma\left\{X_{l, N}^{(\beta)}:(l, \beta) \in I_{N} \backslash B_{k}^{N, R}\right\}\right)\right| .
\end{gathered}
$$

Since we always have

$$
p_{k, N}^{(\alpha)} \leq P\left(V^{(\alpha)}\right)
$$

and (7.10) holds true when $k \in U_{N, n \vee n}$, it follows taking into account (3.2) and (3.4) that

$$
\begin{gathered}
b_{1} \leq K \ell^{2}(1+\psi(n \vee m))^{\ell} R N\left(\left(P\left(V^{(0}\right)\right)^{\ell}+\left(P\left(V^{(1)}\right)\right)^{\ell}\right) \\
\times\left(P\left(V^{(0}\right)+P\left(V^{(1)}\right)\right)+K^{2} \ell^{2}(n \vee m)^{2}\left(P\left(V^{(0}\right)+P\left(V^{(1)}\right)\right)^{2} .
\end{gathered}
$$

In order to estimate $p_{k, l, N}^{\alpha, \beta}$ (and, eventually, $b_{2}$ ) we will essentially repeat the arguments from Section 6. First, observe that

$$
\left(\mathbb{I}_{V^{(0)}} \circ T^{i}\right)\left(\mathbb{I}_{V^{(1)}} \circ T^{j}\right)=0 \text { if }|i-j|<\kappa_{V^{(0)}, V^{(1)}} .
$$

Hence, $p_{k, l, N}^{\alpha, \beta}=0$ if $\delta_{N}(k, l)<\kappa_{V^{(0)}, V^{(1)}}$. Now suppose that $\delta_{N}(k, l)=d \geq$ $\kappa_{V^{(0)}, V^{(1)}}, q_{i_{1}, N}(k) \leq q_{i_{2}, N}(k) \leq \ldots \leq q_{i_{\ell}, N}(k)$ and $q_{j_{1}, N}(l) \leq q_{j_{2}, N}(l) \leq \ldots \leq$ $q_{j_{\ell}, N}(l)$. Assume that the pair $k, l$ does not belong to the exceptional set $D_{N}$ of 
cardinality at most $K$ appearing in Assumption 2.1(iii). Then we have to deal with two alternatives from (6.5).

If the first inequality in (6.5) holds true and $r=q_{i_{1}, N}(k)-q_{j_{1}, N}(l) \geq n \vee m$ then by Lemma 3.2 from [14,

$$
p_{k, l, N}^{\alpha, \beta}=E\left(X_{k, N}^{(\alpha)} X_{l, N}^{(\beta)}\right) \leq(1+\psi(1)) p_{k, N}^{(\alpha)} P\left(V^{(\beta)}\right) .
$$

If, on the other hand, $\kappa_{V^{(0)}, V^{(1)}} \leq r<n \vee m$ then in the same way as in Section 6 we obtain that

$$
p_{k, l, N}^{\alpha, \beta}=E\left(X_{k, N}^{(\alpha)} X_{l, N}^{(\beta)}\right) \leq(1+\psi(1)) P\left(V^{(\alpha)}\right) P\left(T^{n \vee m-r} V^{(\beta)}\right) .
$$

If, in addition, $k \in U_{N, n \vee m}$ then in the same way as in (6.8),

$$
p_{k, l, N}^{\alpha, \beta} \leq(1+\psi(1))^{\ell}\left(P\left(V^{(\alpha)}\right)\right)^{\ell} P\left(T^{n \vee m-r} V^{(\beta)}\right) .
$$

If the second inequality in (6.5) holds true then we obtain (7.16) if $r=q_{j_{\ell}, N}(l)-$ $q_{i_{\ell}, N}(k) \geq n \vee m$ while (7.17) follows if $\kappa_{V^{(0)}, V^{(1)}} \leq r<n \vee m$ and if, in addition, $k \in U_{N, n \vee m}$ then we obtain (7.18). Relying on (3.2)-(3.4), (6.9), (7.10), (7.12), (7.14) and (7.16) -(7.18) we conclude similarly to (6.10) that

$$
\begin{gathered}
b_{2} \leq\left(K^{2} \ell^{3}(1+\psi(1))(n \vee m)\left(P\left(V^{(0)}\right)+P\left(V^{(1)}\right)\right)\right. \\
\left.+K \ell(1+\psi(1))^{\ell} N\left(\left(P\left(V^{(0)}\right)\right)^{\ell}+\left(P\left(V^{(1)}\right)\right)^{\ell}\right)\right)\left(\ell R\left(P\left(V^{(0)}\right)+P\left(V^{(1)}\right)\right)\right. \\
\left.+\sum_{r=\kappa_{V^{(0)}, V^{(1)}}^{n \vee m-1}}^{n}\left(P\left(T^{n \vee m-r} V^{(0)}\right)+P\left(T^{n \vee m-r} V^{(1)}\right)\right)\right) .
\end{gathered}
$$

Similarly to (6.13) we obtain also that

$$
\begin{aligned}
& b_{3} \leq 2^{2 \ell+5} \psi(R-n \vee m)\left(2-(1+\psi(R-n \vee m))^{\ell+1}\right)^{-2} \\
& \times\left(K \ell^{2}(n \vee m)\left(P\left(V^{(0)}\right)+P\left(V^{(1)}\right)\right)\right. \\
&\left.+(1+\psi(1))^{\ell} N\left(\left(P\left(V^{(0)}\right)\right)^{\ell}+\left(P\left(V^{(0)}\right)\right)^{\ell}\right)\right) .
\end{aligned}
$$

These provide the estimate of $A_{2}$ by (7.9), (7.14), (7.15), (7.19) and (7.20).

In order to estimate $A_{3}$ observe that

$$
\begin{gathered}
=\sum_{1 \leq k \leq N, \alpha=0,1}\left|P\left\{Y_{k, N}^{(\alpha)}=1\right\}-P\left\{Z_{k, N}^{(\alpha)}=1\right\}\right| \\
\leq\left(\sum_{k \in U_{N, n \vee m}, \alpha=0,1}+\sum_{1 \leq k \leq N, k \notin U_{N, n \vee m}, \alpha=0,1}\right)\left|P\left\{Y_{k, N}^{(\alpha)}=1\right\}-\left(P\left(V^{(\alpha)}\right)\right)^{\ell}\right| \\
\leq\left((1+\psi(n \vee m))^{\ell}-1\right) N\left(\left(P\left(V^{(0)}\right)\right)^{\ell}+\left(P\left(V^{(1)}\right)\right)^{\ell}\right) \\
\left.+K \ell^{2}(n \vee m)\left(P\left(V^{(0)}\right)+P(1)\right)\right)
\end{gathered}
$$

where in the last inequality we relied on Lemma 3.2 from [14] and on (3.2) above. The estimate of $A_{4}$ we obtain from (17.5),

$$
A_{4} \leq P\left\{\tau^{*}>N\right\}=\left(1-\left(P\left(V^{(1)}\right)\right)^{\ell}\right)^{N}
$$

since we are dealing here with an i.i.d. sequence of Bernoulli random variables. The estimate of $A_{5}$ is the same as in (5.25),

$$
A_{5} \leq 2 P\left(V^{(0)}\right) \text {. }
$$


Finally, combining (7.3)-(7.5), (7.8)-(7.15) and (7.19)-(7.23) we derive (2.15) completing the proof of Theorem 2.8 .

Corollary 2.9 follows from the estimate (2.15) choosing $R=R_{L}=2\left(n_{L} \vee m_{L}\right)$ and if $V_{L}=A_{n_{L}}^{\xi}$ and $W_{L}=A_{m_{L}}^{\eta}$ it remains only to verify the assertion that $\kappa_{A_{n}^{\xi}, A_{m}^{\eta}} \rightarrow \infty$ as $n, m \rightarrow \infty$ provided that $\xi, \eta \in \Omega_{P}$ are not periodic and not shifts of each other. Indeed, $\pi\left(A_{n}^{\xi}\right), \pi\left(A_{m}^{\eta}\right)$ and $\pi\left(A_{n}^{\xi}, A_{m}^{\eta}\right)$ are nondecreasing in $n$ and $m$, and so does $\pi\left(A_{n}^{\xi}, A_{m}^{\eta}\right)$. Hence, the limit $r=\lim _{n, m \rightarrow \infty} \kappa_{A_{n}^{\xi}, A_{m}^{\eta}}$ exists. If $r<\infty$ then, at least, one of the limits $r_{1}=\lim _{n \rightarrow \infty} \pi\left(A_{n}^{\xi}\right), r_{2}=\lim _{m \rightarrow \infty} \pi\left(A_{m}^{\eta}\right)$ or $r_{3}=\lim _{n, m \rightarrow \infty} \pi\left(A_{n}^{\xi}, A_{m}^{\eta}\right)$ is finite. If $r_{1}<\infty$ then $\xi$ is periodic with the period $r_{1}$, if $r_{2}<\infty$ then $\eta$ is periodic with the period $r_{2}$ and if $r_{3}<\infty$ then either $T^{r_{3}} \xi=\eta$ or $T^{r_{3}} \eta=\xi$. Finally, it follows from Lemma 4.1 from [15] that (2.24) holds true for $P \times P$-almost all $(\xi, \eta)$, completing the proof.

\section{REFERENCES}

[1] M. Abadi and B. Saussol, Hitting and returning into rare events for all alpha-mixing processes, Stoch. Process. Appl. 121 (2011), 314-323.

[2] M. Abadi and N. Vergne, Sharp errors for point-wise Poisson approximations in mixing processes, Nonlinearity 21 (2008), 2871-2885.

[3] R. Arratia, L. Goldstein and L. Gordon, Two moments suffice for Poisson approximations: the Chen-Stein method, Ann. Probab. 17 (1989), 9-25.

[4] P.Billingsley, Probability and Measure, 3d ed., J.Willey, New York, 1995.

[5] R. Bowen, Equilibrium States and the Ergodic Theory of Anosov Diffeomorphisms, Lecture Notes in Math. 470, Springer-Verlag, Berlin, 1975.

[6] R.C. Bradley, Introduction to Strong Mixing Conditions, Kendrick Press, Heber City, 2007.

[7] J. Bochnak, H. Coste, M.-F. Roy, Real Algebraic Geometry, Springer, New York, 1998.

[8] M. Demers, P.Wright and L.-S. Young, Entropy, Lyapunov exponents and escape rates in open systems, Ergod. Th.\& Dynam. Sys. 30 (2012), 1270-1301.

[9] Ye. Hafouta, A functional CLT for nonconventional polynomial arrays, arXiv: 1907.03303

[10] L. Heinrich, Mixing properties and central limit theorem for a class of non-identical piecewise monotonic $C^{2}$-transformations, Mathematische Nachricht. 181 (1996), 185214.

[11] Ye. Hafouta and Yu. Kifer, Nonconventional Limit Theorems and Random Dynamics, World Scientific, Singapore, 2018.

[12] Yu. Kifer, Nonconventional Poisson limit theorems, Israel J. Math. 195 (2013), 373-392.

[13] Yu. Kifer, Ergodic theorems for nonconventional arrays and an extension of the Szemerédi theorem, Disc. Cont. Dyn. Sys. 38 (2018), 2687-2716.

[14] Yu. Kifer and A. Rapaport, Poisson and compound Poisson approximations in conventional and nonconventional setups, Probab. Th. Relat. Fields 160 (2014), 797-831.

[15] Yu. Kifer and A. Rapaport, Geometric law for multiple returns until a hazard, Nonlinearity 32 (2019), 1525-1545.

[16] Yu. Kifer and F. Yang, Geometric law for numbers of returns until a hazard under $\phi$-mixing, arXiv: 1812.09927.

[17] K. Petersen, Ergodic Theory, Cambridge Univ. Press, Cambridge, 1983.

[18] I.R. Shafarevich, Basic Algebraic Geometry 1, 3d ed., Springer, Berlin, 1977.

[19] R. Walker, Algebraic Curves, Princeton Univ. Press, Princeton, 1950.

Institute of Mathematics, The Hebrew University, Jerusalem 91904, IsRael

E-mail address: kifer@math.huji.ac.il 